\title{
Neural dynamics of attention switching and temporal-order information in short-term memory
}

\author{
STEPHEN GROSSBERG and GREGORY STONE \\ Center for Adaptive Systems, Boston University, Boston, Massachusetts
}

\begin{abstract}
Reeves and Sperling (1986) have developed an experimental paradigm and a model to explain how attention switching influences the storage of temporal-order information in short-term memory (STM), or working memory. The present article suggests that attention switching influences initial storage of items in STM, but that competitive interactions among the STM representations of stored items control the further evolution of temporal-order information as new items are processed. The laws governing these competitive interactions, called the long-term memory (LTM) invariance principle and the STM normalization rule, were originally derived from postulates that ensure that STM is updated in a way that enables temporally stable list learning in LTM to occur. Despite these adaptive constraints, and often because of them, temporal-order information is not always stored veridically. Both feedforward and feedback STM processes, with different invariant properties, are identified in the storage of temporal-order information.
\end{abstract}

The critical importance of temporary storage in perception has long been recognized (James, 1890) and has been the subject of extensive experimental and theoretical work (Atkinson \& Shiffrin, 1971; Baddeley, 1976; Healy, 1975; Lee \& Estes, 1977, 1981; Rundus, 1971; Sperling \& Melchner, 1976; Tulving, 1983). Such temporary storage occurs at multiple levels of processing, from low-level sensory buffers to conceptually based, cross-modal representations. Although the various forms of temporary storage differ in many details-depending upon the nature of processing at the level in questionthe fundamental role of such storage and the problems that must be solved in devising real-time mechanisms for its instantiation have led to the recognition of several general principles governing the short-term storage process (Grossberg, 1978a, 1978b). Within the framework provided by these principles, it is possible to investigate and specify in greater detail specific short-term storage phenomena. In particular, this paper uses data on the effect of a shift of visual attention on temporal-order information over item representations in short-term memory (Reeves, 1977; Reeves \& Sperling, 1986; Sperling \& Reeves, 1980) to elucidate the dynamics of shortterm memory at a central stage of processing.

This research was supported in part by the Air Force Office of Scientific Research (AFOSR 85-0149), the National Science Foundation (NSF IST-8417756), and the Office of Naval Research (ONR N00014-83K0337). Stephen Grossberg was the primary investigator for all three grants; the latter grant provided full support for Gregory Stone.

We wish to thank Cynthia Suchta for her valuable assistance in the preparation of the manuscript and Adam Reeves for his helpful discussions throughout the development of this work.

S. Grossberg's mailing address is: Center for Adaptive Systems, Boston University, Department of Mathematics, Boston, MA 02215. G. Stone s current mailing address is: Department of Psychology, Arizona State University, Tempe, AZ 85287.

\section{TEMPORAL-ORDER INFORMATION IN SHORT-TERM MEMORY}

A number of paradigms have been used to probe the mechanisms by which subjects can store temporal-order information about a list of items in short-term memory (STM). The present model provides a principled explanation of several of the patterns that have been reported in the literature.

In free-recall tests of short-term memory, items from the beginning and the end of a sufficiently long list are reported earlier than are items from the middle of the list (Atkinson \& Shiffrin, 1971; Murdock, 1962). Although, in general, this bowed serial position effect reflects both long-term memory (LTM) and STM effects, immediate free recall by amnesic subjects with long-term memory deficits also produces a bowed serial position effect (Baddeley \& Warrington, 1970). This suggests that bowing can occur in STM with little or no LTM contribution (Figure 1a). This conclusion is also supported by freerecall data for normal subjects. R. M. Hogan and M. M. Hogan (1975) theoretically disentangled STM and LTM contributions in their free-recall data, and found an STM bow in addition to the expected LTM primacy gradient. Lee and Estes (1981) and Ratcliff (1978) also presented evidence for a primacy effect in STM.

When shorter lists are presented (e.g., the digits of a telephone number), the items can be recalled immediately in the correct order but may be forgotten if a distraction is introduced before recall, suggesting that the correct temporal order is maintained in STM. Correct temporal-order information can be encoded by a primacy gradient in STM (Figure 1b).

Reeves and Sperling (Reeves, 1977; Reeves \& Sperling, 1986) found that when a list is presented rapidly and 

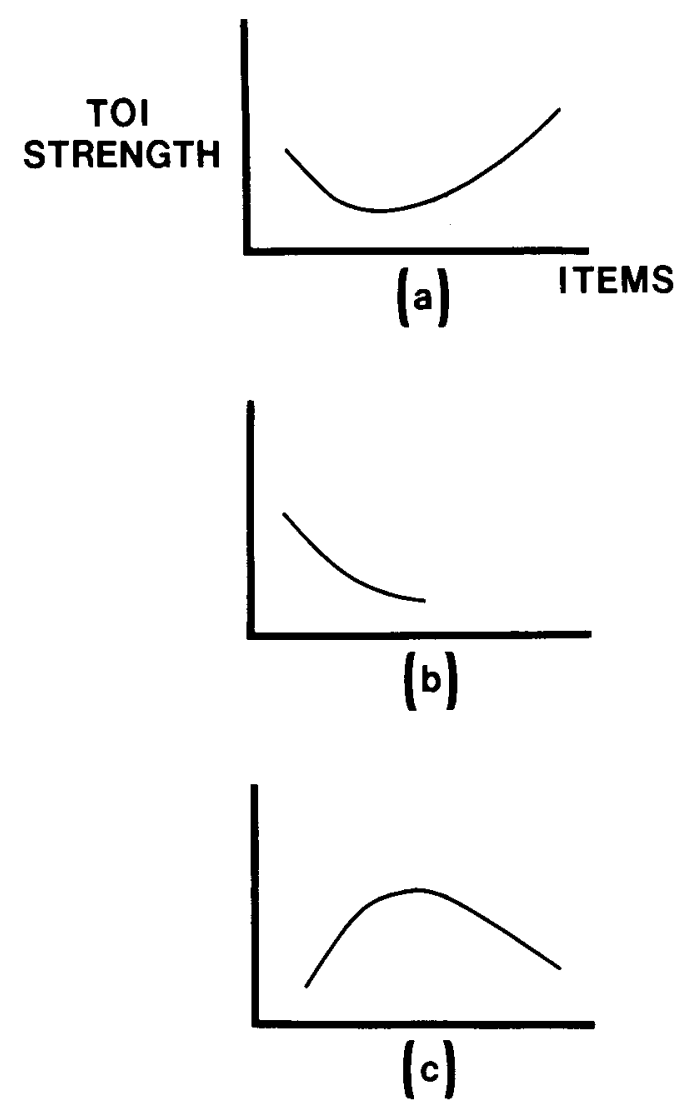

Figure 1. Possible patterns of temporal-order information in STM. The abscissa represents presentation order of items in a list. The ordinate represents the precedence strength of the items, which determines mean report order. (a) Classic bow with primacy for early items and recency for later items; (b) pure primacy giving correct temporal order; (c) inverted bow found by Reeves and Sperling (1986).

subjects must shift visual attention to the list, items in the middle of the list are reported first. Thus, under these conditions, the serial position curve in STM is an inverted bow (Figure 1c).

The type of model that we develop in this paper has already been used to suggest an explanation of primacy gradients and bows in STM (Grossberg, 1978a, 1978b). In this article, we further develop the model to quantitatively analyze the Reeves and Sperling (1986) data. In particular, we consider how attention switching interacts with the processing of temporal-order information in STM during conditions of rapid item presentation. In order to emphasize the critical role that temporal-order information in short-term memory plays in our account of Reeves and Sperling's data, we refer to the model developed in this article as the Temporal-Order Model (TOM). Before presenting the TOM, we will describe the data of Reeves and Sperling, the empirical model that they developed to fit the data, and systematic deviations of their model from the data, which suggest separate effects of attentional gate opening and internal STM dynamics on report order.

\section{THE REEVES-SPERLING PARADIGM}

Reeves and Sperling (1986) had subjects monitor a sequence of letters that appeared successively at a position to the left of a fixation point. The presentation rate of the letters was fixed for each subject so that accuracy in reporting the letters was nearly perfect (about $98 \%$ correct) and the subjects' full attention was required. When a predesignated target symbol was detected, subjects shifted their attention, without moving their eyes, to a stream of numerals that appeared sequentially to the right of the fixation point. Subjects responded by reporting, in order, the first four numerals they were able to detect.

There were two within-subjects variables: target symbol and presentation rate for the numerals. For each of the 3 subjects, the target symbol was either a $\mathrm{C}$, a $\mathrm{U}$, or a square. The choice of target symbol on each trial was randomized and was not known to the subject before the trial began. Two of the subjects (AR and GL), who were experienced in the task, ran in four numeral-presentationrate conditions $(4.6,6.9,9.2$, and 13.4 numerals/second). The third subject (AK), who was naive, ran in three numeral-presentation-rate conditions $(5.6,6.9$, and 9.2 numerals/second). These presentation rates were selected to avoid both "blurring" (about 20 numerals/second) and implicit naming of each numeral as it appeared (about 3 numerals/second; cf. Landauer, 1962; Sperling, 1963). For each subject and presentation rate, the "critical set" was defined as the seven consecutive numerals most likely to be reported, based on pilot studies. Numeral positions were numbered so that the numeral occurring simultaneously with the target symbol was assigned to position 0 . For AR and GL, the critical set began at positions -1 , 0,1 , and 2 for the slowest to the fastest conditions. For $\mathrm{AK}$, the critical set began at positions $-1,1$, and 2 for the slowest to the fastest conditions. Feedback indicating the first six numerals in the critical set was provided after each trial.

Reeves and Sperling (1986) evaluated the data using several performance measures, which can be grouped into item scores and order scores. The item score $P_{i}$ describes the proportion of trials in which the subject reported a numeral from position $i$ in the critical set regardless of its position in the response. Figure 2 shows $P_{i}$ as a function of the onset asynchrony between the target letter and the numeral in position $i$, for each subject in each condition. The bell-shaped curves indicate that subjects most often reported numerals that had been clustered around 400 msec after target onset. Reeves and Sperling also computed $P_{i}(r)$, the proportion of trials in which the numeral in stimulus position $i$ was reported in response to position $r$.

The order score, $P_{i B j}$, describes the proportion of trials on which the numeral in position $i$ was reported before the numeral in position $j$. Typical values of $\boldsymbol{P}_{i B_{j}}$ (Subject AR, Target $U$ ) are presented in Figure 3. 


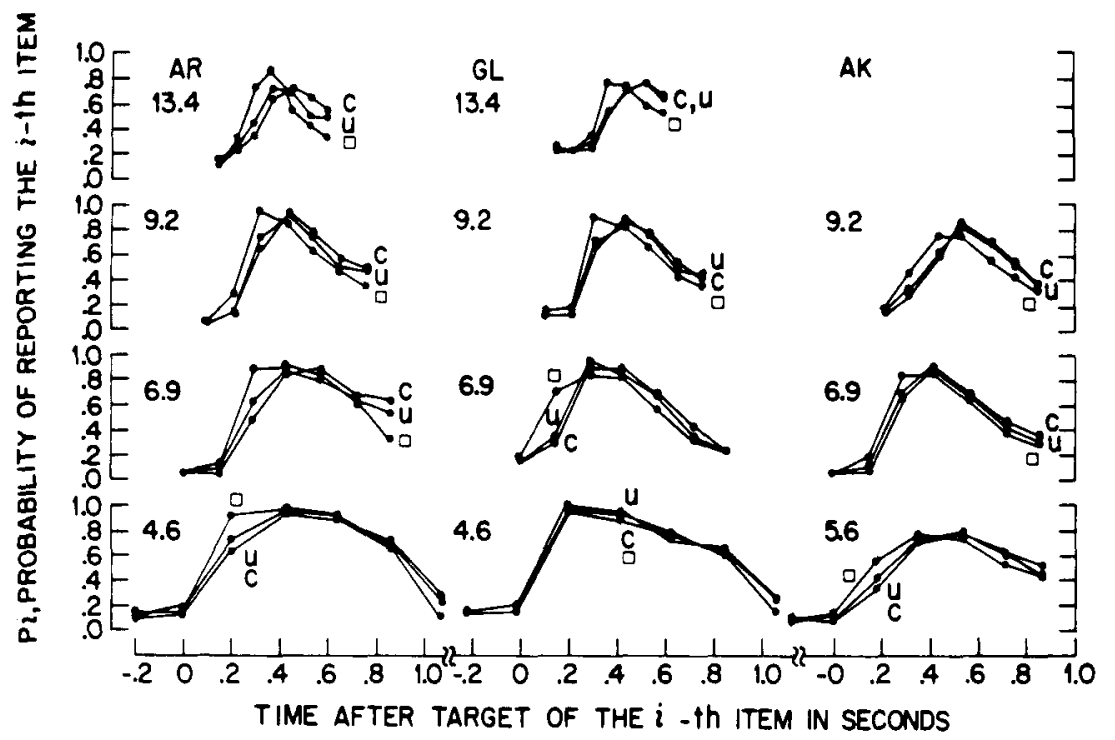

Figure 2. The item score, $P_{i}$, for all conditions in the Reeves and Sperling (1986) experiment. $P_{i}$ describes the proportion of trials in which the subject reports a numeral from position $i$ in the critical set. The abscissa gives the onset asynchrony between the target letter and the numeral in position $i$. Data for a given subject appear in one column, as indicated. Each panel within a column presents the data for the given presentation rate. Each curve within a panel represents a different target condition, as indicated. From "Attentional theory of order information in short-term visual memory" by A. Reeves and G. Sperling, 1986, Psychological Review, 93, p. 184. Reprinted by permission.

When the $P_{i B j}$ curves do not cross each other, they are said to display laminarity. For example, in the upper left quarter of Figure 3 (presentation rate $=13.4$ numerals/ second), stimulus position 6 tends to be reported before all other positions. To the extent that laminarity holds, responses show an order of precedence. In other words, the top curve gives the stimulus position that tends to be reported first, the second curve gives the stimulus position that tends to be reported second, and so forth. Thus, the data for Subject AR, Target U, rate 13.4 numerals/ second shows a precedence order of $(6,5,7,8,4,3,2)$. This pattern of precedence, in which a central position has the greatest precedence and the nearest precentral and postcentral positions are reported next, is referred to as folding. The central position (6 in the preceding example) is called the "folding point." At faster presentation rates, precedence orders showed strong folding. At slower presentation rates, folding was present, but tempered by a tendency to report correct temporal order.

Another aspect of Reeves and Sperling's (1986) results is worth noting. Subjects reported that they actually "saw" the numerals in the reported order and were surprised to find that the reported order was not veridical. This phenomenology was compelling enough that the first author, who also served as a subject, initially assumed that the discrepancy between reported order and the order indicated by the feedback was due to a programming error (A. Reeves, personal communication, 1985). These introspections lead to several conclusions. The re-ordering of numerals in short-term memory occurs prior to conscious awareness. Despite the fact that all list items acti- vate the same retinal region, later items can appear to occur before earlier items without masking recognition of these earlier items. Thus, the later items do not "catch up" to the earlier items to inhibit them. Rather, items are successively transformed from retinal coordinates into a space that can simultaneously store several item representations in short-term, or working, memory. Under certain conditions, later items can encode earlier temporalorder properties than can earlier items. In the TOM, one such temporal-order factor is the relative activation levels of the different item representations in short-term memory (Figure 1). When these item representations are stored in short-term memory, later items can be activated more than earlier items, even though later items began to enter STM after earlier items.

\section{THE REEVES-SPERLING ANALYSIS}

After presenting evidence that discounts both an itemguessing and an order-forgetting account of these results, Reeves and Sperling (1986) established the viability of a one-dimensional scale of precedence for order information in STM for their data. In addition, they assumed that $P_{i B j}$ depends upon the difference in precedences according to the equation:

$$
P_{i B j}=H[V(i)-V(j)],
$$

where $V(i)$ is the precedence strength for stimulus position $i$, and $H$ is some monotone increasing function mapping the reals into the interval $[0,1]$. Reeves and Sperling tested these assumptions using a "quadruple" 

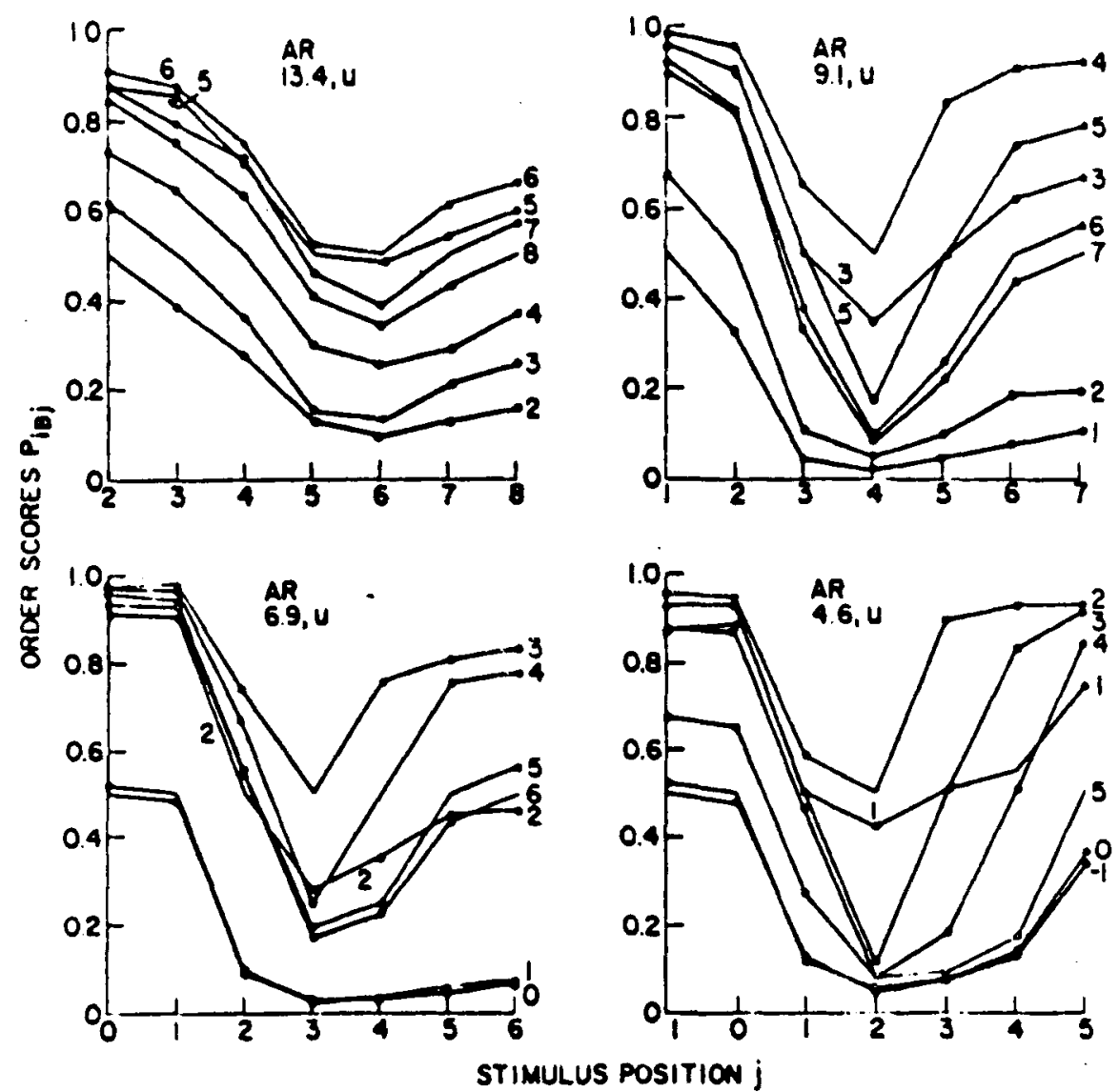

Figure 3. The order score, $\boldsymbol{P}_{i z_{j}}$, for Subject AR, Target U. Quantity $\boldsymbol{P}_{i B_{j}}$ describes the proportion of trials on which the numeral in position $i$ is reported before the numeral in position $j$. The abscissa represents list position $j$. The curve for each value of $i$ is labeled with that value of $i$. The four panels are for the four different presentation rates, as indicated. From "Attentional theory of order information in short-term visual memory" by A. Reeves and G. Sperling, 1986, Psychological Review, 93, p. 187. Reprinted by permission.

condition on the $P_{i B j}$ probabilities (Block \& Marschak, 1960):

$$
P_{i B j}<P_{k B t}<=P_{i B k}<P_{j B !} .
$$

This condition should hold for all $i, j, k$, and $l$ for any choice of the $V(i)$ and $H$ satisfying the constraints previously given. The mean proportion of quadruple violations ranged from $4 \%$ at the fastest rates to $7 \%$ at the slowest rates. Assuming that response order reflects both the precedence strengths $V(i)$ and an equal-variance normally distributed noise component, Reeves and Sperling estimated the seven $V(i)$ for each cell of the subject $\times$ experimental condition design (Figure 4). Each $V(i)$ was derived from Equation 1 by assuming that $H$ was a cumulative normal distribution and then by performing a Monte Carlo simulation.

The inverted- $\mathrm{U}$ shapes of the $V(i)$, based on relative order scores, are quite similar to the $P_{i}$ scores based on the probability of a single item's appearing in the response. In fact, the predicted item scores, using the $V(i)$, accounted for $98 \%$ of the overall variance in the data. For the order scores $P_{i b j}$, the fit was very close at the fast rates but was not as good at slower rates [overall, the $V(i)$ accounted for $94 \%$ of the variance]. Finally, Reeves and Sperling (1986) argued that the violations of laminarity could reflect averaging of trials with different precedence scales and noted that simulations in which trials with the same $V$ functions but different folding points were averaged produced violations of laminarity typical of the data.

\section{THE ATTENTION GATING MODEL (AGM)}

Having demonstrated the utility of the strength model of precedence in accounting for the $P_{i}$ and $P_{i B j}$ scores, Reeves and Sperling (1986) proposed a parsimonious description of precedence strengths using an Attention Gating Model (AGM). The AGM assumes that all stimulus items are represented peripherally and that the strength of temporal-order information in central storage is determined by two factors: the attention gating function $a(t)$, 


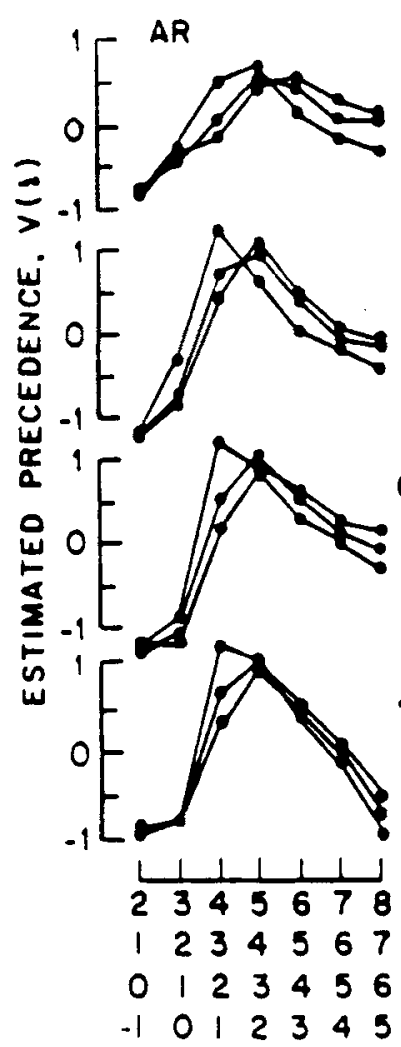

GL

13.4

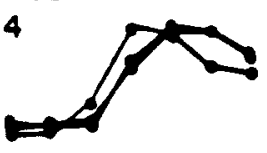

9.2

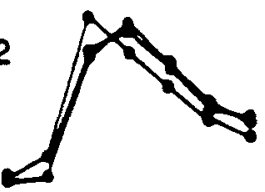

6.9

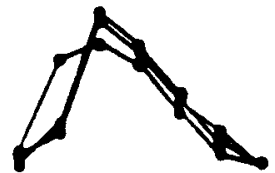

4.6

1234567

01123456

$-10112345$
AK
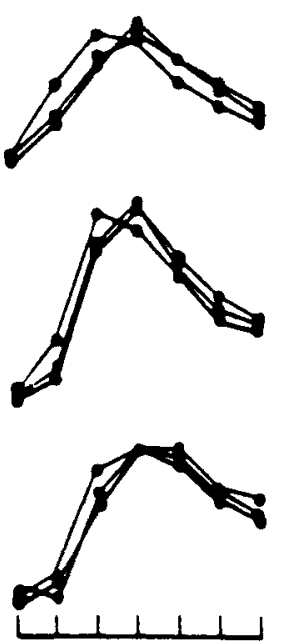

2345678

01253456

$-1012345$

\section{STIMULUS POSITION $i$}

Figure 4. The estimated precedence strengths, $V_{(i)}$, for all experimental conditions: $V_{i i}$ was obtained by least squares fit to the $P_{i B j}$ and $P_{i}(1)$ scores. The abscissa represents position in the critical set. The abscissa labels under the critical set positions give the absolute list positions, with the top row describing the fastest rate and the bottom row the slowest rate. Data for a given subject are presented in one column, as indicated. Each panel within a column presents data for the presentation rate indicated. Each curve in a panel represents a different target condition. From "Attentional theory of order information in short-term visual memory" by $A$. Reeves and G. Sperling, 1986, Psychological Review, 93, p. 191. Reprinted by permission.

and a peripheral availability-or persistence-function, $b_{i}(t)$, for each stimulus position. The STM activity $s_{i}$ for the $i$ th item $r_{i}$ at its time of storage is thus the cumulative central availability of the item's peripheral persistence:

$$
s_{i}=\int_{-\infty}^{\infty} a(w) b_{i}(w) d w .
$$

These $s_{i}$ serve the same function in the AGM that the estimated precedences $V(i)$, as defined in Equation 1, serve for the empirical characterization of Reeves and Sperling's results. Reeves and Sperling assumed that $b_{i}(t)=1$ from the onset of item $r_{i}$ at time $t_{i}$ to the onset of item $r_{i+1}$ at time $t_{i+1}$ and $b_{i}(t)=0$ at all other times. Thus, by Equation 3,

$$
s_{i}=\int_{t_{i}}^{t_{i+1}} a(w) d w .
$$

By Equation 4, an item's STM activity depends entirely upon the degree to which the attention gate is open while the item is being presented. The AGM assumes that there is neither spontaneous STM decay nor any interaction be- tween the STM activities of different item representations. The STM activity of an item at the time of recall is assumed to equal its strength upon completion of central storage.

It is convenient to rewrite Equation 4 in a form that emphasizes that $s_{i}$ is a constant portion of a total integrated STM activity. After $j$ items have been stored in STM, the total STM activity is

$$
T\left(t_{j+1}\right)=\sum_{k=1}^{j} s_{k}=\int_{t_{1}}^{t_{j+1}} a(w) d w .
$$

Using this expression, Equation 4 can be written in the form:

$$
s_{i}=T\left(t_{i+1}\right)-T\left(t_{i}\right) .
$$

In order to compare the $s_{i}$ values with the analogous quantities in the TOM, we define the normalized precedences,

$$
Y_{i}=\frac{T\left(t_{i+1}\right)-T\left(t_{i}\right)}{T(\infty)} .
$$


Equations 3-7 are true within the AGM for any choice of the attention gate $a(t)$. Reeves and Sperling (1986) were able to fit their data by defining $a(t)$ to be a gamma function:

$$
a(t)= \begin{cases}\frac{t^{*}}{\sigma^{2}} e^{-t^{*}} & \text { if } t^{*} \geq 0 \\ 0 & \text { if } t^{*}<0\end{cases}
$$

where

$$
t^{*}=(t-\tau) / \alpha .
$$

In Equation 9, the attentional reaction time, $\tau$, is the time at which the attention gate begins to open; $\alpha$ is a time scale constant; and $\sigma$ scales $a(t)$ relative to the noise distribution. For each subject, $\alpha$ and $\sigma$ were chosen constant across conditions, and a $\tau_{\text {targ }}$ was set for each target letter. For Subject AR, the attentional reaction time, $\tau$ in Equation 8, was dependent only on the choice of target letter, so that $\tau=\tau_{\text {targ }}$. For Subjects GL and AK, the attentional reaction time $\tau$ was assumed to depend upon both the target and the presentation rate. These two factors were assumed to have an independent influence on the attentional reaction time $\tau$. Thus, for these subjects, the $\tau$ in Equation 8 was chosen to satisfy $\tau=\tau_{\text {rarg }}+\tau_{\text {rate }}$, subject to the constraint that $\Sigma_{\tau_{\text {rate }}}=0$ for each subject. The model of the data for Subject GL included three $\tau_{\text {rate }}$ parameters, and that for Subject AK included two $\tau_{\text {rate }}$ parameters. For each subject, a least squares fit to the $P_{i B j}$ and the $P_{i}(1)$ scores was obtained, where $P_{i}(1)$ is the probability that the numeral in stimulus position $i$ will be reported in response position 1 . The fits were quite good for the small number of parameters chosen. The percentage of variance accounted for, by subject, was $83 \%$ for $\mathrm{AR}, 86 \%$ for GL, and $89 \%$ for AK. Thus, 13,200 experimental data points were first reduced to 198 precedence strength parameters, which in turn were replaced by 20 AGM parameters capable of accounting for at least $83 \%$ of the variance in the $P_{i B j}$ and $P_{i}(1)$ performance measures. Clearly, the AGM provides an elegant and parsimonious descriptive model of the experimental results.

However, it is worth noting that comparison of the AGM's predicted precedences, the $Y_{i}$ in Equation 7, with the empirical estimates of precedence, the $V(i)$ in Equation 1, shows distinct trends across presentation order (Figure 5). In particular, the $Y_{i}$ tend to underestimate the $V(i)$ for early items and overestimate them for later items. This difference also tends to become more negative as presentation order approaches the folding point and then becomes more positive as presentation order increases beyond the folding point. In other words, the AGM tends to underestimate the rate of change in precedence scores for early items and overestimate the rate of change for later items. In the AGM, a single parameter, $\alpha$, is used to account for the rate of gate opening and the rate of gate closing; thus, the best fit estimate of $\alpha$ must compromise between the faster rate of change for early items and the slower rate of change for later items.

We will use the descriptive AGM as the basis for an alternative quantitative explanation of the Reeves and

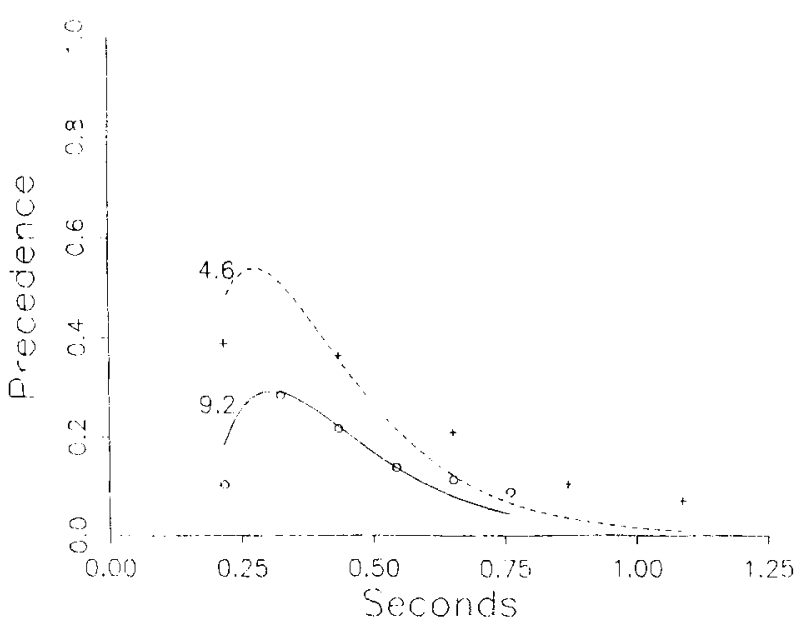

Figure 5. Best fit of normalized AGM precedence scores, $Y_{i}$, to empirically derived precedence scores, $V(i)$. Data are for Subject GL, Target C, rates 9.2 and 4.6 items/second. The abscissa represents each item's presentation order. The curves represent the $Y_{i}$ function and the symbols represent the $V(i)$ scores. Precedence scores for 9.2 items/second are given by the solid curve and the 0 symbols. Precedence scores for the 4.6 items/second condition are given by the dashed curve and the + symbols. Because the $Y_{i}$ and $V(i)$ were scaled differently, a least squares fit of the $Y_{i}$ to the $V(i)$ was performed separately for each condition and used to rescale the $V(i)$.

Sperling (1986) data. This will be done using the TOM, in which STM dynamics between the time of central storage and recall plays a critical role. The principles from which the TOM was derived illustrate some of the reasons we believe it provides a deeper explanation of the Reeves and Sperling data than does the AGM. In particular, the TOM accounts for the decreasing precedence scores for later items in terms of STM dynamics and the increasing precedence of early items as also reflecting the influence of a fast-opening attention gate. This account is consistent with the apparent difference in rate of change of precedence strengths for early and later items, which is not captured by the AGM. In addition, the TOM generalizes to a number of other experimental paradigms in which temporal-order information in STM is critical, whereas the AGM does not. For example, the TOM formulation of STM dynamics has been used to analyze data concerning speech and language recognition (Cohen \& Grossberg, 1986a, 1986b; Grossberg, 1978b, 1986b; Grossberg \& Stone, 1986), free recall (Grossberg, 1978a), serial verbal learning (Grossberg, 1978b), and predictive sequences of motor acts (Grossberg, 1978b, 1986b, 1986c; Grossberg \& Kuperstein, 1986).

\section{THE LTM INVARIANCE PRINCIPLE:}

\section{Deriving STM Laws from an LTM Stability Principle}

We now characterize laws whereby a list of items can be stored in STM. We relate the laws that store individual items in STM to the LTM laws that group, or chunk, these items into unitized lists. We require that individual items 
be stored in STM in a manner that enables LTM to form unitized lists in a temporally stable way. Such laws show how to alter the STM activities of previous items in response to the presentation of new items so that the repatterning of STM activities that is caused by the new items does not inadvertently obliterate the LTM codes for old item groupings.

Consider from this perspective learning of the new word myself, supposing that the words my, self, and elf are already familiar. We would not wish the LTM codes for the words $m y$, self, and elf to be distorted or suppressed just because we are learning the new word myself. On the other hand, the predictive importance of the groupings $m y$, self, and elf may be reduced by their temporal embedding within the list myself. Thus, the laws whereby STM activities over item representations are updated as new items are processed must enable the new items to alter the STM activities of previously processed item groupings without destroying the LTM codes for these groupings. This constraint on STM coding is therefore called the LTM invariance principle (Grossberg, 1978a, 1978b). A remarkable consequence of this principle is that it shows how breakdowns in the encoding of temporalorder information in STM can be derived from mechanisms that enable LTM chunks to be learned in a temporally stable way.

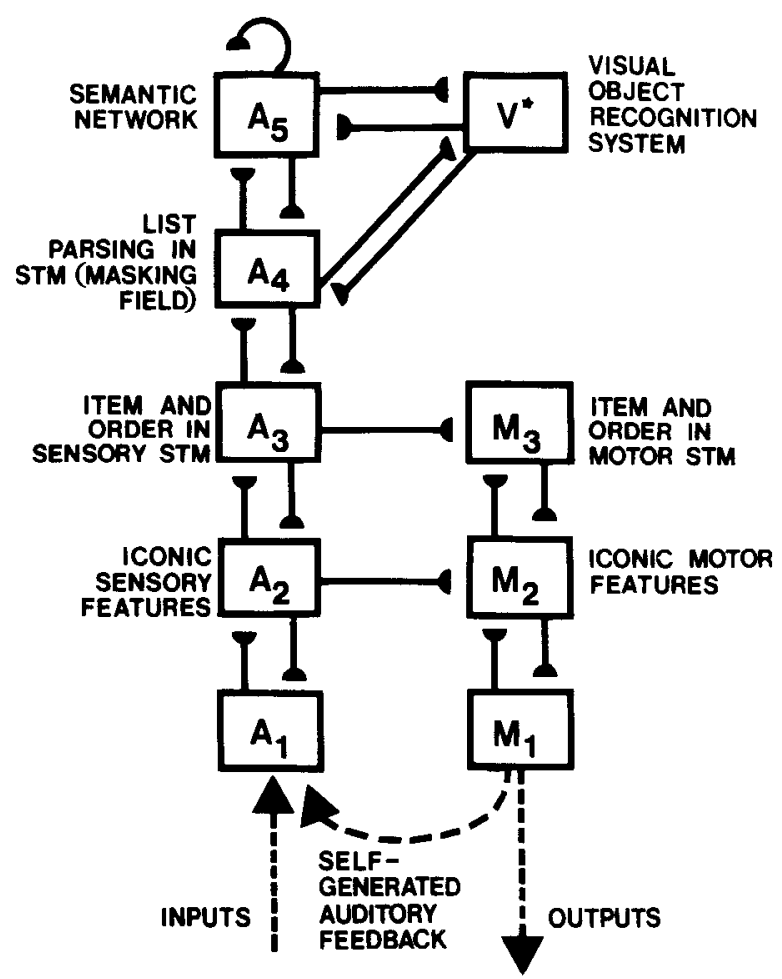

Figure 6. Theoretical macrocircuit involved in processing list items. Stage $A_{3}$ encodes both the identity of items (item information) and a measure of the order in which they were stored (order information). Stage $A_{4}$ encodes unitized sublists of items represented at $A_{3}$ and is called a masking field. From "Neural dynamics of word recognition and recall" by S. Grossberg and G. O. Stone, 1986, Psychological Review, 93, 46-74.
The theoretical macrocircuit depicted in Figure 6 (Cohen \& Grossberg, 1986a; Grossberg, 1978b, 1986b, 1986c; Grossberg \& Stone, 1986) provides a vantage point from which to review briefly how the LTM invariance principle is computationally implemented. In Figure 6, the processing stage $A_{3}$ stores an STM activity pattern that encodes both item information and temporal-order information. Item information is coded in terms of the representations that are activated, whereas temporal-order information is coded in terms of the spatial patterning of activation across representations. In this way, a single spatial pattern of STM activity across $A_{3}$ encodes both the set of items that has been stored (i.e., item information) and a measure-perhaps inaccurate-of the order in which the items were stored (i.e., temporal-order information). As a new item representation in $A_{3}$ is activated by external events, this STM activity pattern is updated to include the new item in a way that satisfies the LTM invariance principle.

Stage $A_{4}$ encodes in STM the sublists, or groupings, of items across $A_{3}$ to which the network is sensitive. In the simplest realization of this idea, a single $A_{4}$ population is activated by an STM pattern across $A_{3}$, and different STM patterns across $A_{3}$ activate different $A_{4}$ populations. In other words, individual $A_{4}$ populations encode whole lists of items. In more general versions of the idea, an STM pattern across $A_{3}$ activates several $A_{4}$ populations. Each $A_{4}$ population encodes a particular sublist of the $A_{3}$ encoding. The spatial patterning of STM activity across these $A_{4}$ populations encodes which sublist groupings of the whole list are salient to $A_{4}$ (in particular, the list as a whole, its most salient parts, and the predictive wholes of which it forms a salient part). A network $A_{4}$ whose STM patterns are capable of representing salient parts and wholes of the $A_{3}$ pattern is called a masking field (Cohen \& Grossberg, 1986a, 1986b, in press).

Both prewired featural biases and subsequent learning experiences help to determine the sublist groupings to which $A_{4}$ is sensitive (Carpenter \& Grossberg, in press a, in press b; Cohen \& Grossberg, in press; Grossberg, 1986b). In particular, the pathways from $A_{3}$ to $A_{4}$ contain modifiable LTM traces that change as a function of the STM patterns that $A_{3}$ experiences. These LTM traces enable $A_{4}$ to react preferentially to familiar sublist groupings. The laws for storing individual items in STM at $A_{3}$ are chosen to guarantee the temporal stability of LTM encoding within the $A_{3} \rightarrow A_{4}$ pathways. We can now state more precisely how the design of $A_{3}$ is constrained by the need to stabilize LTM within the $A_{3} \rightarrow A_{4}$ pathways.

The LTM invariance principle states that the spatial patterns of STM activity across $A_{3}$ are instated and reset by a sequentially presented list in such a way as to leave the $A_{3} \rightarrow A_{4}$ LTM codes of past event groupings invariant.

In order to understand how to implement this rule, it is necessary to know how the $A_{3} \rightarrow A_{4}$ LTM traces help to transform STM activity patterns across $A_{3}$ into STM activity patterns across $A_{4}$. The conditionable pathways from $A_{3}$ to $A_{4}$ define an adaptive filter. In other words, 
if node $v_{i}$ in $A_{3}$ generates a signal $S_{i}$ in the pathway to node $v_{j}$ in $A_{4}$, this signal is multiplied, or gated, by an LTM trace $z_{i j}$ before the gated signal $S_{i} z_{i j}$ can activate $v_{j}$. The sum $T_{j}=\sum_{i} S_{i} z_{i j}$ of all gated signals is the total input from $A_{3}$ to $v_{j}$. The input pattern $\left(T_{1}, T_{2}, \ldots\right)$ determines which sublist groupings will be stored in STM by $A_{4}$. The relative sizes of the signals $S_{i}$ determine the relative sizes of the inputs $T_{j}$. Accordingly, in order to prevent new items at $\boldsymbol{A}_{3}$ from disturbing the LTM coding of past item groupings, we assume that a new item entering $A_{3}$ may alter the absolute sizes of $S_{i}$ for previously active nodes, but does not alter their relative sizes. In other words, each new item can multiply, or shunt, the previous STM activities across $A_{3}$ by a common multiplicative factor as a new item is stored in STM at $A_{3}$. Grossberg $(1978 \mathrm{a}, 1978 \mathrm{~b})$ noted that such a rule could be implemented by designing $A_{3}$ as an on-center (cooperative) off-surround (competitive) recurrent (feedback) network undergoing shunting (mass action) interactions.

In order to implement the LTM invariance principle within the TOM, we consider a sequence of items $r_{1}, r_{2}$, $\ldots, r_{n}$ presented at times $t_{1}, t_{2}, \ldots, t_{n}$, respectively. We work with the STM activities $x_{i}\left(t_{j+1}\right)$ for the representation of item $i$ that obtain just before the $r_{j+1}$ item begins to take effect. The LTM invariance principle constrains the development, during the course of list presentation, of these STM activities. The STM activities at time $t_{j+1}$ (the instant that item $j+1$ begins to take effect) provide the best estimate of the equilibrated pattern of STM activity in response to the $j$ th item in the list. We introduce the notation $\mu_{i}=x_{i}\left(t_{i+1}\right)$ for the STM activity of the $i$ th item at the time its STM memory trace is first activated. Quantity $\mu_{i}$ covaries with the amount of attention paid to the $i$ th item in the list at the time it is presented, with smaller $\mu_{i}$ reflecting less attention. The theory sharply distinguishes between the vigilance devoted by a subject to a stimulus and the amount of attention received by that stimulus in STM. See Carpenter and Grossberg (1986, in press b) and Grossberg and Stone (1986) for discussions of this distinction. We also introduce the notation $\omega_{j}$ for the STM reset parameter, which multiplies, or shunts, the STM traces of all previous items upon entry of the $j$ th item into STM. These relationships are expressed mathematically by the equation

$$
x_{i}\left(t_{j+1}\right)= \begin{cases}0 & \text { if } i>j \\ \mu_{i} & \text { if } i=j \\ \omega_{j} x_{i}\left(t_{j}\right) & \text { if } i<j\end{cases}
$$

Thus, the activity of each item's STM representation is the product of a term, $\mu_{i}$, reflecting the attention paid to each item as it is presented and of shunting parameters, $\omega_{j}$, which reset STM when each subsequent item is presented. Recursive solution of Equation 10 leads to an equation for $x_{i}\left(t_{j+1}\right)$ expressed entirely in terms of these factors:

$$
x_{i}\left(t_{j+1}\right)=\mu_{i} \prod_{k=i+1}^{j} \omega_{k}, \quad i \leq j
$$

Although each $\mu_{i}$ reflects attentional factors and will vary according to the task, we can specify the STM reset parameters $\omega_{j}$ in terms of the attentional parameters $\mu_{i}$.

\section{THE NORMALIZATION RULE: Limited Capacity of STM}

Expression of the STM reset parameters, $\omega_{j}$, in terms of the attentional weights, $\mu_{i}$, is achieved by implementing the STM normalization rule. The STM normalization rule refers to the fact that total STM strength grows toward a finite maximum level of activity that is independent of the number of items being processed. In other words, STM at the item level $A_{3}$ is of limited capacity. This rule is not an independent assumption within the theory. Rather, it is a property of the competitive STM interactions among the item representations that also give rise to the LTM invariance principle (Grossberg, 1978a, $1978 \mathrm{~b})$. In order to impose the STM normalization rule, we first derive a convenient equation for the total STM activity, $S\left(t_{j+1}\right)$, after the item field has equilibrated in response to storage of the $j$ th item.

By definition,

$$
S\left(t_{j+1}\right)=\sum_{i=1}^{j} x_{i}\left(t_{j+1}\right)
$$

Using Equation 10 of the LTM invariance principle, Equation 12 implies that

$$
S\left(t_{j+1}\right)=\omega_{j} S\left(t_{j}\right)+\mu_{j} .
$$

Equation 13 suggests a functional relationship between the STM reset parameters $\omega_{j}$ and the attentional parameters $\mu_{i}$. This relationship must satisfy three requirements: (1) If $\mu_{j}=0$, then $\omega_{j}=1$ : If the attentional weight $\mu_{j}$ for item $j$ equals zero (i.e., item $j$ receives no attention and so produces no STM activity), the total STM activity should not change due to that item. (2) $\omega_{j}$ is a decreasing function of $\mu_{j}$ : As the attentional weight $\mu_{j}$ is parametrically increased, the STM reset weight $\omega_{j}$ is parametrically decreased. In other words, the greater the attentiona] weight $\mu_{j}$ given to the item currently being stored, the greater its ability to competitively reset the STM traces of previous items. This implies that as the attentiona weight $\mu_{j}$ increases, previous item codes will be mors strongly inhibited. (3) Given constant vigilance across ex. perimental trials, $\omega_{j}$ covaries with $\mu_{j}$ in such a way that $S\left(t_{j+1}\right)$ increases monotonically to a finite limit as succes. sive items are stored.

\section{RELATING STM RESET TO ATTENTIONAL PROCESSING}

Our computations herein have implemented the simples! function that satisfies these constraints:

$$
\omega_{j}=1-\mu_{j}
$$

where $0 \leq \mu_{j} \leq 1$. This choice allows us to derive ex- 


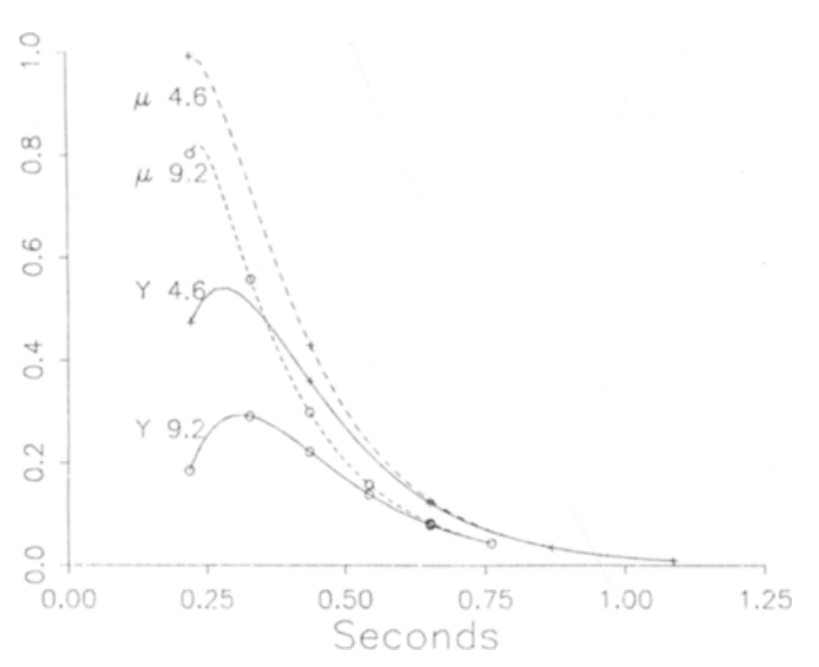

Figure 7. Comparison of TOM attentional weights $\mu_{i}$ and normalized AGM precedence scores $Y_{i}$. Data shown are for Subject GL, Target C, rates 9.2 and 4.6 items/second. In the TOM, each item's strength at storage is given by the $\mu_{i}$; however, due to STM reset, its strength on retrieval from STM is given by the $Y_{i}$. In the AGM, each item's strength does not change after storage and is given by the $Y_{1}$. The abscissa represents presentation order. The solid curves represent the $Y_{i}$ scores and the dashed curves represent the $\mu_{i}$ scores. The 4.6 items/second condition is indicated by the + symbols. The 9.2 items/second condition is indicated by the $\circ$ symbols.

plicit attentional weights $\mu_{j}$ from the precedence scores predicted by the AGM.

Before implementing these computations, it is necessary to verify that Equation 14 implies the STM normalization rule. By Equations 13 and 14,

$$
S\left(t_{j+1}\right)=\left(1-\mu_{j}\right) S\left(t_{j}\right)+\mu_{j},
$$

from which it follows that

$$
S\left(t_{j+1}\right) \leq 1, j \geq 0 .
$$

By rewriting Equation 15 as

$$
1-S\left(t_{j+1}\right)=\left(1-\mu_{j}\right)\left[1-S\left(t_{j}\right)\right],
$$

it is also readily seen that $S\left(t_{j+1}\right)$ increases with $j$ as long as $0 \leq \mu_{j} \leq 1$ for all $j=1,2, \ldots$ By Equations 14 and 17 ,

$$
\frac{1-S\left(t_{j+1}\right)}{1-S\left(t_{j}\right)}=\omega_{j}
$$

By Equation 18, the rate $\omega_{j}$, with which previously active representations are reset, as in Equation 13, equals the rate with which previously uncommitted STM capacity becomes committed. Thus, the linear relationship (Equation 14) describes the important special case in which a balance exists between the processes of STM resource utilization and STM reset.

Equations 11 and 14 allow us to express the activity of each item in STM as a function of the attentional weights assigned to the items as they were presented:

$$
x_{i}\left(t_{j+1}\right)=\mu_{i} \prod_{k=i+1}^{j}\left(1-\mu_{k}\right)
$$

In the preceding analysis, we considered two fundamental constraints on STM processing, the LTM invariance principle and the STM normalization rule, which result from an analysis of how a processing system can learn in a stable fashion in response to a temporal list of events. These principles allow us to convert a given sequence of attentional weights, which characterize each item's strength upon storage in STM, into an expanding spatial pattern of STM activities that define the precedence scores responsible for report order at the time of response. In the following section, we consider the reverse problem: how to convert estimated precedences based on observed responses into estimated sequences of attentional weights. Further analysis of these empirically derived attentional weight sequences will then reveal theoretically interesting characteristics of the interaction between STM dynamics and attentional gating processes. In particular, conversion of the AGM precedence scores into our formulation reveals an unanticipated interaction between feedforward and feedback processes in the storage of temporal-order information in STM.

\section{DERIVATION OF ATTENTIONAL WEIGHTS FROM THE AGM}

Equation 19 converts a sequence of attentional weights (the $\mu_{i}$ ) into a set of temporal-order precedence scores [the $\left.x_{i}\left(t_{j+1}\right)\right]$. The analysis of experimental results requires conversion of observed response orders into unobserved attentional weights. The model, as developed thus far, can be used for this analysis without additional assumptions.

We begin by relating the normalized precedence values $Y_{i}$ of the AGM, which are defined in Equation 7, to the analogous quantities within the TOM. If $n$ is the number of items in the list, then $x_{i}\left(t_{n+1}\right)$ is the STM activity of item $r_{i}$ at the time of read out from STM, assuming that spontaneous STM decay is not appreciable. We therefore let

$$
x_{i}\left(t_{n+1}\right)=Y_{i}
$$

as a basis for deriving further information about the TOM from the AGM. First, we compute the attentional weights as a function of AGM parameters.

\section{Theorem 1}

The attentional weights $\mu_{i}$ are a function of the onset asynchrony $\delta$ between successive items, the time $\tau$ at which the attention gate begins to open, and the rate parameter $\alpha$ in Equation 8:

$$
\mu_{i}=\frac{\left(1+t_{i}^{*}\right) e^{-t_{i}^{*}}-\left(1+t_{i+1}^{*}\right) e^{-t_{i+1}^{*}}}{1-\left[\left(1+t_{i+1}^{*}\right) e^{-t_{i+1}^{*}}-\left(1+t_{n+1}^{*}\right) e^{-t_{n+i}^{*}}\right]},
$$


where

$$
t_{i}^{*}=\left\{\begin{array}{cl}
\frac{\delta i-\tau}{\alpha} & \text { if } \delta i>\tau \\
0 & \text { if } \delta i \leq \tau
\end{array}\right.
$$

The proof is given in Appendix A.

Figure 7 compares the sequence of these $\mu_{i}$ values with the $Y_{i}$ at each presentation rate, using the data for Subject GL, Target $\mathrm{C}$.

Unlike the pattern of precedences $Y_{i}$, the pattern of attentional weights $\mu_{i}$ does not exhibit significant bowing. In other words, bowing is not a major effect within the TOM at the times when items are first stored in STM. The TOM is thus consistent with the existence of an attention gate that opens more rapidly than does the gate hypothesized in the AGM. Since $x_{i}\left(t_{n+1}\right)=Y_{i}$, the TOM suggests that much of the bowing effect evolves due to competitive interactions that reset the STM activities of item representations when later items are presented. Competitive interactions also account for the decrement in the attentional weights $\mu_{i}$ for later items $r_{i}$.

\section{STM ONSET AND STM RESET}

The distinct effects of attention switching and of STM interactions on temporal-order information can be better seen through the STM onset equation,

$$
x_{i}\left(t_{j}\right)=\gamma v_{i} x_{i-1}\left(t_{j}\right), j>i,
$$

where

$$
\gamma=e^{\frac{-\delta}{\alpha}}
$$

and the STM reset equation,

$$
x_{i}\left(t_{j+1}\right)=\omega_{j} x_{i}\left(t_{j}\right), j>i .
$$

The STM onset equation describes the relative activities, $x_{i}\left(t_{j}\right) / x_{i-1}\left(t_{j}\right)$, of the STM representations corresponding to successive items $r_{i-1}$ and $r_{i}$ at all times $t_{j}$ after the items are stored in STM. The equation says that these relative activities equal a quantity $\gamma \nu_{i}$ that is independent of $t_{j}$. In other words, the relative advantage or disadvantage of items $r_{i-1}$ and $r_{i}$ in STM is determined by the time $r_{i}$ is stored and does not change thereafter.

The STM reset equation describes the relative sizes, $x_{i}\left(t_{j+1}\right) / x_{i}\left(t_{j}\right)$, of the STM activities corresponding to a single item, $r_{i}$, at successive times $t_{j}$ and $t_{j+1}$ after the item is stored in STM. The equation says that these relative activities equal a quantity, $\omega_{j}$, that is independent of the item $r_{i}$. In other words, after items are stored in STM, a new item resets all their stored activities by a constant multiple, $\omega_{j}$. The STM reset equation realizes the LTM invariance principle, as in Equation 10.

The meaning of Equations 23 and 25 resides within two gain functions, $\gamma \nu_{i}$ and $\omega_{i}$, whose forms are derived by fitting Equations 23 and 25 to the AGM. Such a fit discloses some remarkable and unexpected properties. Before describing these properties, we introduce a physical interpretation of Equations 23 and 25 which the properties are used to support and refine.
Function $\gamma \nu_{i}$ is called the feedforward gain function and $\omega_{i}$ is called the feedback gain function. Function $\gamma \nu_{i}$ determines a property of STM activities as items begin to be stored. We suggest that this function reflects, among other processes, the opening of an attention gate. Function $\omega_{i}$, in contrast, determines a property of STM activities after they are stored. We suggest that this function reflects interactions between STM activities that are already stored. The following properties of these functions support these interpretations: Function $\nu_{i}$ is (1) a decreasing function of $i$; (2) greater than 1; and (3) shift invariant in item time. Function $\omega_{i}$ is (1) an increasing function of $i$; (2) less than 1 ; and (3) shift invariant in real time.

Examples of the functions $\nu_{i}$ and $\omega_{i}$, as derived from fitting Equations 23 and 25 to the TOM's fit to the data of Subject AR, Target U, are plotted in Figure 8. Figure 8 also plots each function in real time $(t)$ and in item time $(i)$ to demonstrate the shift invariance described by property (3).

Equations 23 and 25 can be better understood by writing them in a notation that more explicitly exhibits their dependent variables. Since $t_{j}=\delta j$, where $\delta$ is the onset asynchrony, Equation 23 can be rewritten as

$$
x_{i}(\delta j)=e^{\frac{-\delta}{\alpha}} N(i, \tau, \alpha, \delta) x_{i-1}(\delta j)
$$

where

$$
\nu_{i} \equiv N(i, \tau, \alpha, \delta)
$$

and

$$
x_{i}(\delta(j+1))=\Omega(j, \tau, \alpha, \delta) x_{i}(\delta j),
$$

where

$$
\omega_{j} \equiv \Omega(j, \tau, \alpha, \delta) .
$$

\section{OPENING OF THE ATTENTION GATE}

Figure 9 schematizes how the shift invariance in item time of $N(i, \tau, \alpha, \delta)$ interacts with $e^{\frac{-\delta}{\alpha}}$ to control whether correct temporal-order information or folding will occur. By Equation 26, correct temporal-order information (an STM primacy gradient) occurs for items $r_{i-1}$ and $r_{i}$ such that

$$
e^{\frac{-\delta}{\alpha}} N(i, \tau, \alpha, \delta)<1 .
$$

Reversed temporal-order information (an STM recency gradient) occurs for items $r_{i-1}$ and $r_{i}$ such that

$$
e^{\frac{-\delta}{\alpha}} N(i, \tau, \alpha, \delta)>1 \text {. }
$$

Folding occurs if the feedforward gain function $e^{\frac{-\delta}{\alpha}} N(i, \tau, \alpha, \delta)$ passes through the value 1 at an index $i>1$.

As presentation rate decreases, the onset asynchrony $\delta$ increases. Consequently, $N$ shifts to the left and $e^{\frac{\delta}{\alpha}}$ increases, yielding curves such as those in Figure 9. By Equation 30, primacy occurs if $N<e^{\frac{\delta}{\alpha}}$; by Equation 31 , recency occurs if $N>e^{\frac{\delta}{\alpha}}$. Figure 9 indicates 

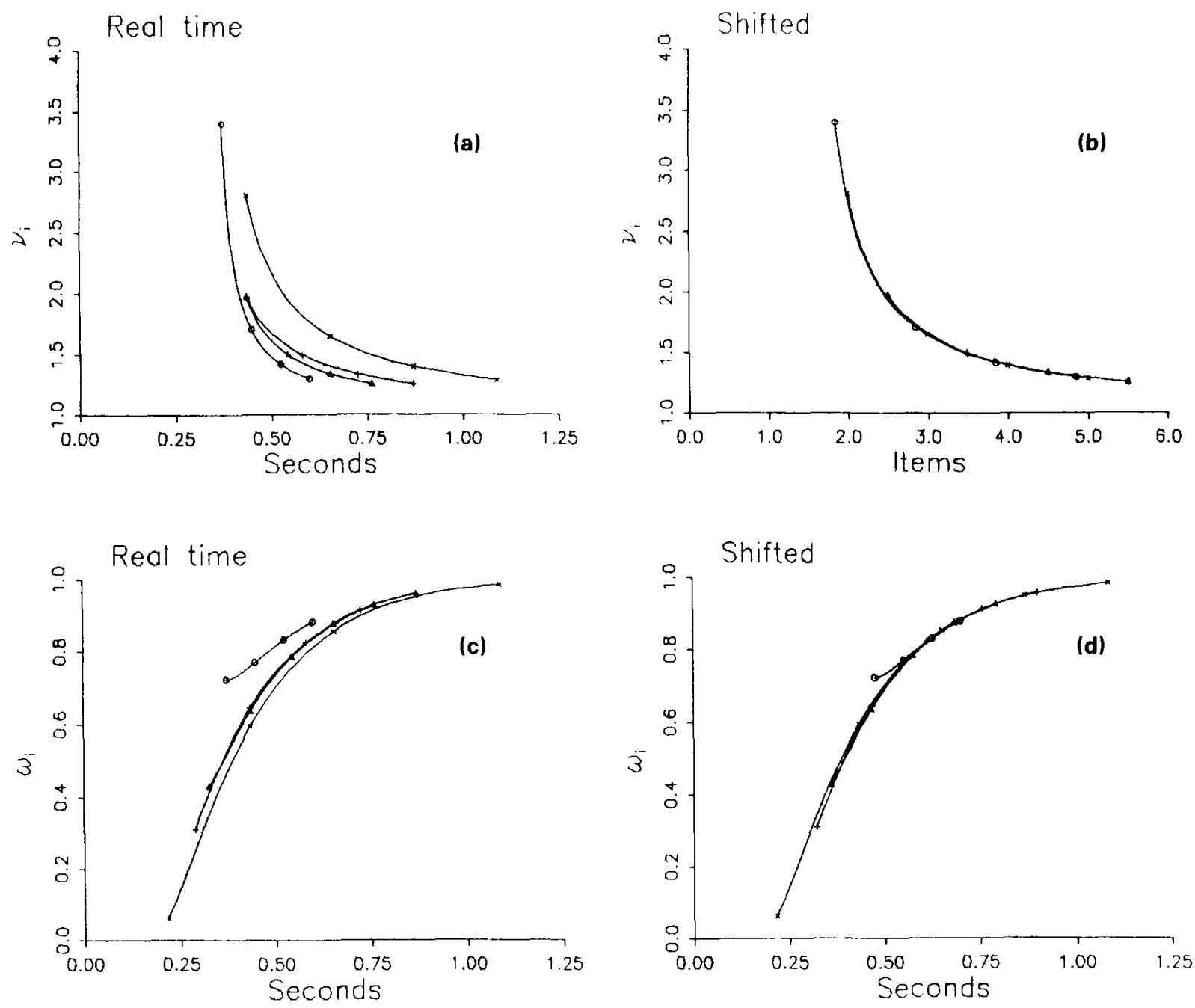

Figure 8. The feedforward gain, $\nu_{i}$, feedback gain, $\omega_{i}$, and their shift invariance properties. All data are for Subject AR, Target U. The four curves in each graph represent the four presentation rate conditions. (a) The feedforward gain function, $\nu_{i}$, is plotted against the onset asynchrony measured in seconds. (b) The $\nu_{i}$ functions are plotted against onset asynchrony measured in number of items elapsed (item time) and have been shifted onto the curve for 4.6 items/second. (c) The feedback gain function, $\omega_{i}$, is plotted against the onset asynchrony measured in seconds. (d) The $\omega_{i}$ have been shifted in real time onto the curve for 4.6 items/second.

that the folding point moves toward the beginning of the list as $\delta$ increases. When it is smaller than the first item to be processed, correct temporal-order information prevails.

The shift invariance of $N$ in item time implies that processing of items is attenuated until a later list position when $\delta$ is small. The term $\gamma=e^{\frac{-\delta}{\alpha}}$ describes the effect of an integration process that takes place in real time within the duration $\delta$ between successive items. More items can be crowded into a constant duration when $\delta$ is small than when $\delta$ is large. Correspondingly, $\gamma^{-1}=e^{\frac{\delta}{\alpha}}$ in Figure 9 decreases as $\delta$ decreases, with the effect that brief presentation of many items in a fixed duration tends to cause folding. In effect, the STM activities of early items relative to later items are weakened if they are presented very quickly.

This effect may be related to the opening of an attention gate by considering the limiting case in which $N \cong 1$ for all items. In other words, choose $\delta$ so large that $N \cong 1$ before even the first item's STM storage equilibrates. Then Equation 23 reduces to

$$
x_{i}\left(t_{j}\right) \cong \gamma x_{i-1}\left(t_{j}\right)
$$

for all items, which shows that correct temporal-order information obtains if gate opening does not prevent full storage of all the items. The proof that $\nu_{i}$ is shift invariant in item time is provided in Appendix B. Function $\nu_{i} \equiv$ $N(i, \tau, \alpha, \delta)$ is a function of $i$ and the variables $\alpha / \delta$ and $\tau / \delta$, which are the parameters $\alpha$ and $\tau$ scaled in item time:

$$
N(i, \tau, \alpha, \delta)=\frac{i-1-P\left(\frac{\tau}{\delta}, \frac{\alpha}{\delta}\right)}{i-P\left(\frac{\tau}{\delta}, \frac{\alpha}{\delta}\right)},
$$

where

$$
P(u, v)=u-v-\frac{1}{1-e^{-\frac{1}{v}}} .
$$




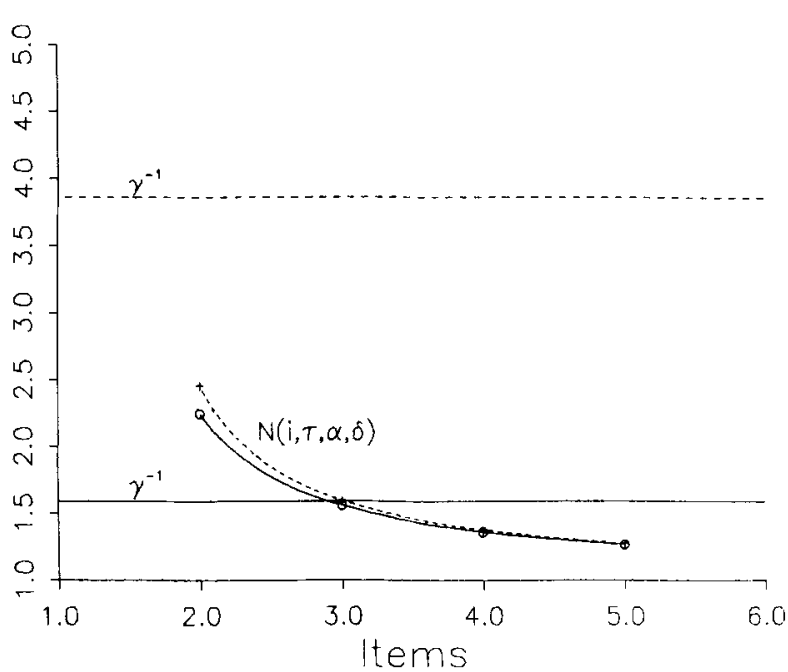

Figure 9. Illustration of the interaction of $N$ and $\gamma^{-1}$ to determine folding versus correct temporal order. When $N$ is greater than $\gamma^{-1}$, a recency effect obtains. When $N$ is less than $\gamma^{-1}$, a primacy effect obtains. Thus, the intersection of $N$ and $\gamma^{-1}$ indicates the point at which folding occurs. For slow presentation rates (dashed curves), $\gamma^{-1}$ is large and passes over the $N$ curve without intersecting it. This implies correct report of temporal order and no folding. For fast presentation rates (solid curves), $\gamma^{-1}$ is smaller and intersects the $N$ curve, so that folding occurs with the item immediately preceding the intersection (in this case the second item) as the folding point.

\section{STM RESET MEDIATED BY INTER-ITEM FEEDBACK INTERACTIONS}

Equation 25 and its more precise counterpart, Equation 28, describe the reset of STM that is driven by new items competing for limited-capacity STM resources. This conclusion can be supported in several ways:

The gain $\Omega$ in Equation 28 increases toward 1 as the item $j$ increases. The explicit equation for $\Omega$ is derived in Appendix A. When $j \gg 1$, STM is hardly reset by a new item, because the limited capacity of STM is almost fully utilized. The approximate shift invariance of $\Omega(j, \tau, \alpha, \delta)$ in real time (Figure 8) also clarifies the STM reset interpretation. Shift invariance implies that for every pair of onset asynchronies $\left(\delta_{1}\right.$ and $\left.\delta_{2}\right)$, there exists a shift $(\lambda)$ such that

$$
\Omega_{\delta_{1}}(t-\lambda) \cong \Omega_{\delta_{2}}(t)
$$

for all times $t$ at which both functions are defined. Consider a time $T$ that can be written in the form $T=j_{1} \delta_{1}$ $=j_{2} \delta_{2}$ for appropriate choices of indices $j_{1}$ and $j_{2}$. In particular, suppose $\delta_{1}<\delta_{2}$ and $j_{1}>j_{2}$. Then we can define

$$
\Omega \equiv \Omega_{\delta_{1}}(T-\lambda) \cong \Omega_{\delta_{2}}(T) .
$$

Hence, by Equation 28,

$$
x_{i}\left(\delta_{1}\left(j_{1}+1\right)-\lambda\right)=\Omega x_{i}\left(\delta_{1} j_{1}-\lambda\right)
$$

and

$$
x_{i}\left(\delta_{2}\left(j_{2}+1\right)\right)=\Omega x_{i}\left(\delta_{2} j_{2}\right)
$$

Equations 37 and 38 say that, up to a shift in time scale, a fixed amount, $\Omega$, of STM reset is determined by continuous STM integration for the duration $T$, whether this processing has been divided among many items, each integrated for a brief time as in Equation 37, or few items, each integrated for a longer time, as in Equation 38. When many items are presented quickly, each item can reset previous items less, but the cumulative effect of storing a fixed total STM activity is to generate a fixed amount of reset. Thus the STM reset equation embodies a type of Bloch's Law trade-off between number of items and processing time to describe how cumulative STM activity regulates subsequent STM storage and reset.

This interpretation is strengthened by consideration of the hypothesis

$$
\mu_{j}=1-\omega_{j}
$$

that was made in Equation 14. As $\omega_{j}$ increases toward 1, less STM reset can occur. Correspondingly, by Equation 39, new items cannot be well stored in STM.

A more detailed interpretation of Equation 39 can be given by noting a remarkable property of the $\mu_{j}$ functions that was empirically derived from the data. We believe that this property provides additional evidence for the neural existence of the $\mu_{j}$ process, and thus for the STM reset process that converts $\mu_{j}$ values into $x_{j}$ values.

In particular, the following significant linear trend was found for later items in the critical set:

$$
\ln \mu_{j}=-m_{\delta} j+b_{\delta} \text {. }
$$

The slopes $m_{\delta}$ and $b_{\delta}$ can themselves be fit as linear functions of the onset asynchrony $\delta$ :

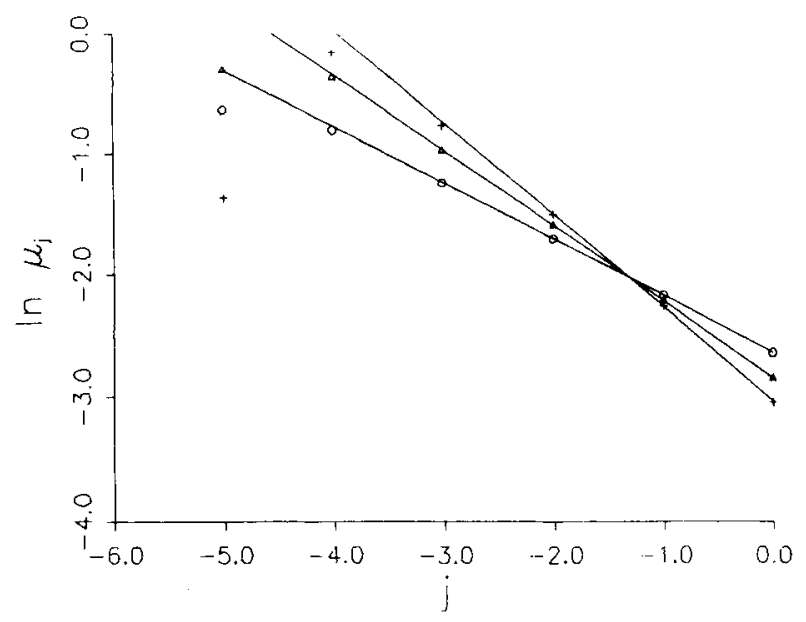

Figure 10. $\ln \mu_{j}$ as a function of $\boldsymbol{j}$ (presentation order). Data are for Subject AK, target square. Best fit regression lines, based on the final three items in each presentation rate condition, are compared with the actual $\ln \mu_{j}$ values, represented by the symbols. Each regression line is labeled with the presentation rate, in items/second, of the condition it represents. The symbols representing the $\ln \mu_{j}$ are assigned to presentation rate as follows: $\circ, 5.6$ items/second; $\triangle$, 8.9 items/second; +, 9.2 items/second. A detailed description of the regression analysis for these conditions is given in Table 3. 


$$
m_{\delta}=m \delta-c,
$$

and

$$
b_{\delta}=b \delta-d,
$$

where $c \cong 0$. Setting $c=0$, we find that

$$
\ln \mu_{j} \simeq-m \delta j+b \delta-d .
$$

Illustrative plots of estimated $\ln \mu_{j}$ values against $j$ are shown in Figure 10. A detailed description of the regressions is provided in Appendix C. Deviations from this log linear function are more pronounced for early items and faster rates, and so are consistent with the opening of an attention gate. Note that, at larger values of $\delta$, the first significantly attended item whose $\mu_{j}$ is stored in STM tends to be larger. In other words, this item has more time in which its activity can be integrated in STM. On the other hand, the slope $-m \delta$ of $\ln \mu_{j}$ as a function of $j$ tends to be steeper at larger values of $\delta$. Thus, a fixed number of items can interfere more with subsequent STM storage of the next item if each of these items has had more time to be stored in STM.

\section{STM ONSET AND STM RESET INTERACT TO DETERMINE TEMPORAL-ORDER INFORMATION}

The nature of the interaction between STM onset and reset factors in determining temporal-order information may be seen by combining Equations 10,23 , and 25 to show that

$$
\mu_{i}=\gamma \nu_{i} \omega_{i} \mu_{i-1} .
$$

Equation 44 follows by letting $j=i+1$ in Equation 23:

$$
x_{i}\left(t_{i+1}\right)=\gamma \nu_{i} x_{i-1}\left(t_{i+1}\right)
$$

rewriting Equation 25 as

$$
x_{i-1}\left(t_{i+1}\right)=\omega_{i} x_{i-1}\left(t_{i}\right) ;
$$

combining Equations 45 and 46 to find

$$
x_{i}\left(t_{i+1}\right)=\gamma \nu_{i} \omega_{i} x_{i-1}\left(t_{i}\right)
$$

and using Equation 10 to rewrite Equation 47 as Equation 44.

Equation 44 shows that the processes that cause successive items $r_{i-1}$ and $r_{i}$ to generate different activities $\mu_{i-1}$ and $\mu_{i}$ when the items are first stored in STM include both STM onset factors $\gamma \nu_{i}$ (notably attentional gating) and STM reset factors $\omega_{i}$ (notably competitive feedback interactions among active STM representations). The STM reset mechanism synchronizes the STM activities of successive items by transforming $\mu_{i-1}$ into the STM activity $x_{i-1}\left(t_{i+1}\right)=\omega_{i} \mu_{i-1}$ one time step later. The STM onset mechanism in Equation 45 shows how attentional gating when $\mu_{i}$ is being stored can cause further differences in the STM activities of successive items.

\section{COMPARISON WITH THE GAGM MODEL}

This article suggests how attentional processes can modulate the temporal unfolding of competitive interactions among active STM representations. The combined effects of both processes give rise to temporal-order information in STM that may not veridically encode the external order of item presentation.

In our analysis of the Reeves and Sperling (1986) data, we have fit the TOM parameters to the AGM in order to analyze whether, indeed, opening of an attention gate, combined with competitive shunting interactions among active STM representations, can provide a conceptually better explanation of these data than can the AGM. Several analytic results strongly support this conclusion. These include the unexpected discovery of the feedforward gain function $\gamma \nu_{i}$ and the feedback gain function $\omega_{i}$, the unexpected discovery of the shift-invariant properties of these functions, the confirmation of the expected property that the attention function $\mu_{i}$ is monotone decreasing rather than bowed, and the unexpected discovery of the Bloch's Law trade-off between number of items and processing time that is shown by the form of $\mu_{i}$ in Equation 40 as a function of experimental parameters.

In addition to their analysis of the AGM, Reeves and Sperling (1986) also sketched a Generalized Attention Gate Model (GAGM) that is closer in spirit to the TOM. As in the TOM, the GAGM postulated that order information is regulated by a shunting competitive interaction. Using the notation introduced herein, the GAGM mixes item and order information via the equation

$$
x_{i}=\mu_{i}\left(1-\beta \sum_{k=1}^{i-1} \mu_{k}\right)
$$

rather than via the TOM equation

$$
x_{i}\left(t_{j+1}\right)=\mu_{i} \prod_{k=i+1}^{j} \omega_{k} .
$$

Thus, the GAGM supplements the item measure $\mu_{i}$ with the competitive order measure $\beta \mu_{i} \sum_{k=1}^{i-1} \mu_{k}$. Unlike in the TOM, in the GAGM, once the activity level $x_{i}$ of item $i$ is established, it is not updated as additional items are stored in STM. Thus, the GAGM assumes that each of the activities $\mu_{i}$ is independently stored in STM, after which all stored items compete in a feedforward fashion to determine the activities $x_{i}$ at the next processing stage. In contrast, the TOM posits that item representations are activated at a previous processing stage before interacting with each other at the next processing stage via a feedback competitive interaction. This feedback process enables both previously stored activities and newly presented items to update their STM activities by struggling for a limited-capacity total STM resource.

One major difference between the models is that a recency gradient in STM, as in a free-recall paradigm, 
can only be generated by the GAGM if successive activities $\mu_{i}$ increase to offset the growing effect of the accumulating inhibition $\beta \mu_{i} \sum_{k=1}^{i-1} \mu_{k}$. This would seem to require that increasing attention be paid to later list items to generate a recency gradient in a free-recall study, which seems not to be the case. In contrast, a feedback competitive interaction can generate a recency gradient in STM without making this assumption (Grossberg, 1978a, 1982). The invariance properties and the Bloch's Law properties derived from the TOM also seem to argue for a feedback competitive process in the control of temporalorder information. In any case, both models point to the importance of shunting competitive processes in the regulation of temporal-order information, and future analyses will doubtless disclose finer details than either model has yet articulated within this general class of competitive mechanisms.

As indicated above, in other applications of the TOM, where the form and time scale of attention switching are not themselves the processes under analysis, the hypothesis that constant attention is paid to stimulus materials does not lead to the folding of temporal-order information through time. Instead, Grossberg (1978a, 1978b, 1982) analyzed situations in which a recency gradient in STM, a bow in STM (Figure 1a), or a primacy gradient in STM (Figure 1b) are the only outcomes that can occur. Moreover, under these circumstances, the list position at which the STM activity pattern begins to bow does not change as more list items are presented. This strong property is a consequence of the LTM invariance principle (see the section entitled "STM Onset and STM Reset"). Thus, one can define the transient memory span of a list to be the list position at which an STM bow begins to develop in a given experimental setup. Measurement of the transient memory span is to be desired in paradigms that provide relatively pure measures of STM processing. In general, measurement of the transient memory span is complicated by the fact that STM and LTM processes often interact, as in generating the bowed curve observed in free-recall experiments (Atkinson \& Shiffrin, 1971; Baddeley \& Warrington, 1970; Hogan \& Hogan, 1975). Circumstances have been characterized during which the transient memory span is shorter than the more familiar immediate memory span. A model of STM and LTM interactions has also been developed wherein the contribution of both types of factors to the observed temporal-order information can, in principle, be disentangled.

In the present article, we have assumed that each item code is represented by a single population activity $x_{i}(t)$ through time, since the data do not probe the internal organization of an item's STM representation. Such concepts as the LTM invariance principle easily generalize to consideration of item representations that are distributed among several unequally activated populations. In this general case, each new item again shunts the STM activity of all previously activated populations at level $A_{3}$ without regard to which combinations of these popula- tions possess unitized list codes at the next processing level $A_{4}$ (see the section entitled "The LTM Invariance Principle").

Breakdowns of temporal-order information in STM are traced in all these cases to biological processes that possess a manifest adaptive value. The competitive STM interactions are designed to enable stored STM patterns to be stably encoded in LTM. Factors such as attentional gating have also been related to stabilization of LTM encoding (Carpenter \& Grossberg, 1986, in press a, in press b; Grossberg, 1980, 1984). The TOM thus suggests that even experimental paradigms that provide relatively pure measures of STM processing, such as the remarkably flexible and informative Reeves and Sperling paradigm, indirectly probe brain mechanisms that are designed to enable us to learn about the world in a stable fashion. This insight suggests the value of developing new experimental paradigms in which relatively pure measures of STM processing can be taken before and after subjects are trained to achieve different stages or types of learning. Such direct manipulations of STM and LTM interactions may provide important new information concerning the adaptive principles that govern brain design.

\section{REFERENCES}

Atkinson, R. C., Shiffrin, R. M. (1971, August). The control of short term memory. Scientific American, 225, 82-90.

BADDELEY, A. D. (1976). The psychology of memory. New York: Basic Books.

Baddeley, A. D., \& Warrington, E. K. (1970). Amnesia and the distinction between long- and short-term memory. Journal of Verbal Learning \& Verbal Behavior, 9, 176-189.

BLoCK, H. D., \& MARSCHAK, J. (1960). Random orderings and stochastic theories of response. In I. Olkin, S. Gharye, W. Hoeffding, W. Madow, \& H. Mann (Eds.), Contributions to probability and statistics. Stanford: Stanford University Press.

Carpenter, G. A., \& Grossberg, S. (1986). Neural dynamics of category learning and recognition: Structural invariants, reinforcement, and evoked potentials. In M. L. Commons, S. M. Kosslyn, \& R. J. Herrnstein (Eds.), Pattern recognition and concepts in animals, people, and machines. Hillsdale, NJ: Erlbaum.

Carpenter, G. A., \& Grossberg, S. (in press a). A massively parallel architecture for a self-organizing neural pattern recognition machine. Computer Vision, Graphics, \& Image Processing.

Carpenter, G. A., \& Grossaerg, S. (in press b). Neural dynamics of category learning and recognition: Attention, memory consolidation, and amnesia. In J. Davis, R. Newburgh, \& E. Wegman (Eds.), Brain structure, learning, and memory. AAAS Symposium Series.

Cohen, M. A., \& Grossberg, S. (1986a). Neural dynamics of speech and language coding: Developmental programs, perceptual grouping, and competition for short term memory. Human Neurobiology, 5, 1-22.

Cohen, M. A., \& Grossberg, S. (1986b). Unitized recognition codes for parts and wholes: The unique cue in configural discriminations. In M. L. Commons, S. M. Kosslyn, \& R. J. Herrnstein (Eds.), Pattern recognition and concepts in animals, people, and machines. Hillsdale, NJ: Erlbaum.

Cohen, M. A., \& Grossberg, S. (in press). Masking fields: A massively parallel neural architecture for learning, recognizing, and predicting multiple groupings of patterned data. Applied Optics.

GROSSBERG, S. (1978a). Behavioral contrast in short-term memory: Serial binary memory models or parallel continuous memory models? Joumal of Mathematical Psychology, 17, 199-219.

GRosSBERG, S. (1978b). A theory of human memory: Self-organization and performance of sensory-motor codes, maps, and plans. In 
R. Rosen \& F. Snell (Eds.), Progress in theoretical biology (Vol. 5, pp. 233-374). New York: Academic Press.

Grossberg, S. (1980). How does a brain build a cognitive code? Psychological Review, 87, 1-51.

GrossberG, S. (1982). Studies of mind and brain: Neural principles of learning, perception, development, cognition, and motor control. Boston: Reidel Press.

Grossberg, S. (1984). Some psychophysiological and pharmacological correlates of a developmental, cognitive, and motivational theory. In R. Karrer, J. Cohen, \& P. Tueting (Eds.), Brain and information: Event related potentials (pp. 58-151). New York: New York Academy of Sciences.

Grossberg, S. (1986a). The adaptive brain. I: Cognition, learning, reinforcement, and rhythm. Amsterdam: Elsevier/North-Holland.

Grossberg, S. (1986b). The adaptive brain. II: Vision, speech, language, and motor control. Amsterdam: Elsevier/North-Holland.

GrossBerg, S. (1986c). The adaptive self-organization of serial order in behavior: Speech, language, and motor control. In E. C. Schwab \& H. C. Nusbaum (Eds.), Pattern recognition by humans and machines. Vol. I: Speech perception (pp. 187-294). New York: Academic Press.

Grossberg, S., \& KUPERSTEIN, M. (1986). Neural dynamics of adaptive sensory-motor control: Ballistic eye movements. Amsterdam: Elsevier/North-Holland.

GrossberG, S., \& StONE, G. O. (1986). Neural dynamics of word recognition and recall: Attentional priming, learning, and resonance. Psychological Review, 93, 46-74.

HeAly, A. F. (1975). Separating item from order information in shortterm memory. Journal of Verbal Learning \& Verbal Behavior, 13, 644-655.

Hogan, R. M., \& Hogan, M. M. (1975). Structural and transient components of memory. Memory \& Cognition, 3, 210-215.

JAMES, W. (1890). The principles of psychology. New York: Holt.

Landauer, T. K. (1962). Rate of implicit speech. Perception \& Motor Skills, 15, 646.

LEE, C., \& EsTES, W. K. (1977). Order and position in primary memory for letter strings. Joumal of Verbal Learning \& Verbal Behavior, 16, 395-418.

LEE, C. , \& ESTES, W. K. (1981). Item and order information in shortterm memory: Evidence for multilevel perturbation processes. Journal of Experimental Psychology: Human Learning \& Memory, 7 , 149-169.

MURDOCK, B. B., JR. (1962). The serial position effect in free recall. Journal of Experimental Psychology, 64, 482-488.

Ratcliff, R. A. (1978). A theory of memory retrieval. Psychological Review, 85, 59-108.

REEVES, A. (1977). The identification and recall of rapidly displayed letters and digits. Unpublished doctoral dissertation, City University of New York.

ReEves, A., \& Sperling, G. (1986). Attentional theory of order information in short-term visual memory. Psychological Review, 93, 180-206.

RuNDUS, D. (1971). Analysis of rehearsal processes in free recall. Journal of Experimental Psychology, 89, 63-77.

SperLING, G. A. (1963). A model for visual memory tasks. Human Factors, 51, 19-31.

Sperling, G., \& Melchner, M. J. (1976). Estimating item and order information. Journal of Mathematical Psychology, 13, 192-213.

SPerLiNG, G., \& ReEves, A. (1980). Measuring the reaction time of a shift of visual attention. In R. Nickerson (Ed.), Attention and performance VIII. Hillsdale, NJ: Erlbaum.

Tulving, E. (1983). Elements of episodic memory. Oxford: Clarendon Press.

\section{APPENDIX A}

Computation of Attention Weights and STM Reset Weights

Theorem 1 (Equations 21 and 22) expresses the attentional weights $\mu_{i}$, as a function of the AGM parameters $\delta, \alpha, \tau$ :

$$
\mu_{i}=\frac{\left(1+t_{i}^{*}\right) e^{-t_{i}^{*}}-\left(1+t_{i+1}^{*}\right) e^{-t_{i+1}^{*}}}{1-\left[\left(1+t_{i+1}^{*}\right) e^{-t_{i+1}^{*}}-\left(1+t_{n+1}^{*}\right) e^{\left.-t_{n+1}^{*}\right]}\right.},
$$

where

$$
t_{i}^{*}=\left\{\begin{array}{cc}
\frac{\delta i-\tau}{\alpha} & \text { if } \delta i>\tau \\
0 & \text { if } \delta i \leq \tau .
\end{array}\right.
$$

The proof of Theorem 1 begins by equating the precedence scores in the two models at the time of STM readout (Equation 20):

$$
x_{i}\left(t_{n+1}\right)=Y_{i} .
$$

We next observe that, due to the LTM invariance principle, the ratio of successive item strengths is unaffected by STM reset, so that

$$
\frac{Y_{i}}{Y_{i+1}}=\frac{x_{i}\left(t_{n+1}\right)}{x_{i+1}\left(t_{n+1}\right)}=\frac{x_{i}\left(t_{i+2}\right)}{x_{i+1}\left(t_{i+2}\right)}=\frac{\omega_{i+1} \mu_{i}}{\mu_{i+1}},
$$

where the final equality derives from the formal statement of the LTM invariance principle (Equation 10). By introducing the assumed relationship,

$$
\omega_{i}=1-\mu_{i},
$$

from Equation 14, Equation A4 provides a recursive relationship between the $\mu_{i}$, using the $Y_{i}$ :

$$
\mu_{i}=\frac{Y_{i}}{Y_{i+1}} \frac{\mu_{i+1}}{\left(1-\mu_{i+1}\right)} .
$$

To convert this recursive relationship into a direct expression for the $\mu_{i}$ in terms of the $Y_{j}$ alone, we exploit the fact that the final item in the list is not reset by subsequent item storage, so that:

$$
Y_{n}=x_{n}\left(t_{n+1}\right)=\mu_{n} .
$$

With $i=n-1$ in Equation A6, we can derive the expression for $\mu_{n-1}$ using Equation A6:

$$
\mu_{n-1}=\frac{Y_{n-1}}{Y_{n}} \cdot \frac{Y_{n}}{\left(1-Y_{n}\right)}=\frac{Y_{n-1}}{1-Y_{n}} .
$$

The general expression for $\mu_{i}$ in terms of the $Y_{i}$ begins to emerge when the expression for $\mu_{n-1}$ (Equation A8) is substituted into Equation A6 to give

$$
\mu_{n-2}=\left(\frac{Y_{n-2}}{Y_{n-1}}\right)\left(\frac{Y_{n-1}}{1-Y_{n}}\right)\left(\frac{1}{1-\frac{Y_{n-1}}{1-Y_{n}}}\right) \text {. }
$$

Multiplying the third term by $\left(1-Y_{n}\right) /\left(1-Y_{n}\right)$, then cancelling the $1-Y_{n}$ and $Y_{n-1}$ terms that appear in the numerator and denominator, reduces Equation A9 to

$$
\mu_{n-2}=\frac{Y_{n-2}}{1-Y_{n-1}-Y_{n}} .
$$

We now prove by induction that

$$
\mu_{i}=\frac{Y_{i}}{1-\sum_{j=i+1}^{n} Y_{j}} .
$$

By Equation A10, this is true for the initial condition $i=n-2$. Using Equation A6, we now show that the case for $\mu_{i-1}$ follows from the case of $\mu_{i}$ : 


$$
\mu_{i-1}=\left(\frac{Y_{i-1}}{Y_{i}}\right)\left(\frac{Y_{i}}{1-\sum_{j=i+1}^{n} Y_{j}}\right)\left(\frac{1}{1-\frac{Y_{i}}{1-\sum_{j=i+1}^{n} Y_{j}}}\right) .
$$

Multiplying the third term by $\left(1-\sum_{j=i+1}^{n} Y_{j}\right) /\left(1-\sum_{j=i+1}^{n} Y_{j}\right)$ and cancelling the $Y_{i}$ and $1-\sum_{j=i+1}^{n} Y_{j}$ terms that appear in the numerator and denominator, we obtain

$$
\mu_{i-1}=\frac{Y_{i-1}}{1-\sum_{j=i+1}^{n} Y_{j}-Y_{i}}=\frac{Y_{i-1}}{1-\sum_{j=i}^{n} Y_{j}},
$$

which is Equation A11 for the case $\mu_{i-1}$.

Note that the relationship between $\mu_{i}$ and the $Y_{j}$ given in Equation A11 derives from the LTM invariance principle and the relationship between $\mu_{i}$ and $\omega_{i}$ given by Equation A5. Equation A11 will hold for any arbitrary set of $Y_{j}$. In other words, Equation A11 does not compute the pattern of temporal-order information in STM at the time of readout. Rather, it provides a method for converting an arbitrary set of observed precedence scores into a sequence of attentional weights, $\mu_{i}$.

To finish the proof of Theorem 1 , we need only substitute the precedence scores $Y_{j}$ derived from the AGM into Equation A11. Setting the attention gate function, $a(\omega)$, in Equation 5 equal to the gamma function assumed by Reeves and Sperling (Equations 8 and 9) gives $Y_{i}$ as

$$
Y_{i}=\int_{t_{i}^{*}}^{t_{i+1}^{*}} \omega e^{-\omega} d \omega=-\left.(1+\omega) e^{-\omega}\right|_{t_{i}^{*}} ^{t_{t+1}^{*}}
$$

so that

$$
Y_{i}=\left(1+t_{i}^{*}\right) e^{-t_{i}^{*}}-\left(1+t_{i+1}^{*}\right) e^{-t_{i+1}^{*}}
$$

with $t_{i}^{*}$ defined as in Equation A2. Likewise,

$$
\sum_{j=i+1}^{n} Y_{j}=\left(1+t_{i+1}^{*}\right) e^{-t_{i+1}^{*}}-\left(1-t_{n+1}^{*}\right) e^{-t_{n+1}^{*}}
$$

Theorem 1 (Equation A1) follows from substitution of Equations A15 and A16 into Equation A11.

Using Equation A5, $\omega_{i}$ can also be expressed in terms of the AGM parameters as a corollary of Theorem 1. By Equation A1 and Equation A5,

$$
\omega_{i}=1-\frac{\left(1+t_{i}^{*}\right) e^{-t_{i}^{*}}-\left(1+t_{i+1}^{*}\right) e^{-t_{i+1}^{*}}}{1-\left[\left(1+t_{i+1}^{*}\right) e^{-t_{i+1}^{*}}-\left(1+t_{i+1}^{*}\right) e^{-t_{n+1}^{*}}\right]},
$$

which implies

$$
\omega_{i}=\frac{1-\left[\left(1+t_{i}^{*}\right) e^{-t_{i}^{*}}-\left(1+t_{n+1}^{*}\right) e^{-t_{n+1}^{*}}\right]}{1-\left[\left(1+t_{i+1}^{*}\right) e^{-t_{i+1}^{*}}-\left(1+t_{n+1}^{*}\right) e^{-t_{n+1}^{*}}\right]} .
$$

where

and

$$
P(\xi, \eta)=\xi-\eta-\frac{1}{1-e^{-\frac{1}{\eta}}}
$$

$$
\begin{aligned}
\xi & =\frac{\tau}{\delta}, \\
\eta & =\frac{\alpha}{\delta} .
\end{aligned}
$$

Shift invariance follows from Equation B1 as follows. Let one or more of the parameters $\tau, \alpha, \delta$ (especially $\delta$ ) be changed to new values $\tau^{*}, \alpha^{*}, \delta^{*}$, so that $P(\xi, \eta)$ is replaced by $P\left(\xi^{*}, \eta^{*}\right)$ in Equation B1. Then given any fixed value $\nu_{i}=\nu$, the indices

$$
i=\frac{1}{y-1}+P(\xi, \eta)
$$

and

$$
i^{*}=\frac{1}{\nu-1}+P\left(\xi^{*}, \eta^{*}\right)
$$

corresponding to the different parameter choices shift by an amount

$$
i-i^{*}=P(\xi, \eta)-P\left(\xi^{*}, \eta^{*}\right)
$$

that is independent of $\nu$. In other words, a change in the parameters $\tau, \alpha, \delta$ shifts the entire curve in item time by the predicted amount $P(\xi, \eta)-P\left(\xi^{*}, \eta^{*}\right)$. From Equation B1, the equation

$$
\nu_{i}=N(i, \tau, \alpha, \delta)=\frac{i+1-P\left(\frac{\tau}{\delta}, \frac{\alpha}{\delta}\right)}{i-P\left(\frac{\tau}{\delta}, \frac{\alpha}{\delta}\right)}
$$

(Equation 33) follows immediately.

The inverse function (Equation $\mathrm{B} 1$ ) is derived from the definition (Equation 23) of $\nu_{i}$ :

$$
\gamma \nu_{i}=\frac{x_{i}\left(t_{j}\right)}{x_{i-1}\left(t_{j}\right)}, j>i,
$$

where

$$
\gamma=e^{\frac{-\delta}{\alpha}}
$$

Letting $j=n+1$ in Equation $\mathrm{B} 9$ and using Equation 20 to convert into the AGM precedences $Y_{i}$, we obtain:

$$
\gamma \nu_{i}=\frac{Y_{i}}{Y_{i-1}} .
$$

By Equation A15 of Appendix A,

$$
\gamma \nu_{i}=\frac{\left(1+t_{i}^{*}\right) e^{-t_{i}^{*}}-\left(1+t_{i+1}^{*}\right) e^{-t_{i+1}^{*}}}{\left(1+t_{i-1}^{*}\right) e^{-t_{i-1}^{*}}-\left(1+t_{i}^{*}\right) e^{-t_{i}^{*}}}
$$

The definition of $t_{i}^{*}$ in Equation 22 implies that

$$
t_{i+1}^{*}=\frac{\delta}{\alpha}+t_{i}^{*}
$$

and thus that

$$
e^{-t_{i+1}^{*}}=e^{\frac{-\delta}{\alpha}} e^{-t_{i}^{*}}=\gamma e^{-t_{i}^{*}} .
$$


Using Equations B13 and B14, all terms $t_{i+1}^{*}$ and $t_{i-1}^{*}$ in Equation B12 can be expressed in terms of $t_{i}^{*}$. Then cancelling the $e^{-t_{i}^{*}}$ terms from the numerator and denominator leads to

$$
\gamma \nu_{i}=\frac{\left(1+t_{i}^{*}\right)-\left(1+t_{i}^{*}+\frac{\delta}{\alpha}\right) \gamma}{\left(1+t_{i}^{*}-\frac{\delta}{\alpha}\right) \gamma^{-1}-\left(1+t_{i}^{*}\right)}
$$

Further simplification is achieved by combining the $1+t_{i}^{*}$ terms on the right and dividing the left and right sides of Equation B15 by $\gamma$ :

$$
\nu_{i}=\frac{\left(1+t_{i}^{*}\right)(1-\gamma)-\frac{\delta \gamma}{\alpha}}{\left(1+t_{i}^{*}\right)(1-\gamma)-\frac{\delta}{\alpha}}
$$

Rearranging Equation B16 to get all $t_{i}^{*}$ terms on the left-hand side leads to

$$
\left(1+t_{i}^{*}\right)(1-\gamma)\left(\nu_{i}-1\right)=\frac{\delta}{\alpha}\left(\nu_{i}-\gamma\right)
$$

By dividing both sides of Equation B17 by $(1-\gamma)\left(\nu_{i}-1\right)$, rewriting the term $\nu_{i}-\gamma$ as $\nu_{i}-1+1-\gamma$, and dividing wherever possible, we obtain

$$
t_{i}^{*}=\frac{\delta}{\alpha}\left(\frac{1}{\nu_{i}-1}-\frac{1}{1-\gamma}\right)-1 .
$$

Finally, we substitute for $t_{i}^{*}$ using Equation 22 and move all terms on the left except $i$ to the right:

$$
i=\frac{1}{\nu_{i}-1}+\frac{\tau}{\delta}-\frac{\alpha}{\delta}-\frac{1}{1-\gamma},
$$

which is the same as the inverse function in Equation B1.

\section{APPENDIX C \\ Computation of ln $\mu_{j}$ Regressions}

Because the attention gate introduces deviation from the $\log$ linear $\mu$ functions, regressions were performed for the final three items in each condition, where attention gating has the least impact.

The index $j$ is based on an item's position relative to the final item in the list. This indexing method has several advantages. First, indexing from the first item to receive attention is complicated by the possibility that this first item can receive so little attention that it has essentially no impact on subsequent item storage and is virtually undetectable in noise (cf. condition AR, Target $U$, rate 13.4 in Table 1). In other words, indices anchored at the first attended item are sensitive to decisions about how much attention constitutes "significant" activation of an item's code. Second, when the indices are anchored at the final item in the list, a significant linear trend of the intercept, $b_{\delta}$, as a function of onset asynchrony, $\delta$, emerges. This is not the case when the indices are anchored at the first attended item.

Tables 1-3 present the regression analyses for subjects AR, $\mathrm{GL}$, and $\mathrm{AK}$, respectively. For each target $\times$ presentation rate condition, $\mu, \ln \mu$ and the best fit to $\ln \mu$, denoted by $\ln \hat{\mu}$, are given for each item. The regression equation, correlation coefficient, and significance level are given for each condition. The regressions of $m_{s}$ and $b_{\delta}$, as a function of onset asynchrony, are given for each target condition.

Table 1

Subject AR, Target $\mathbf{U}$

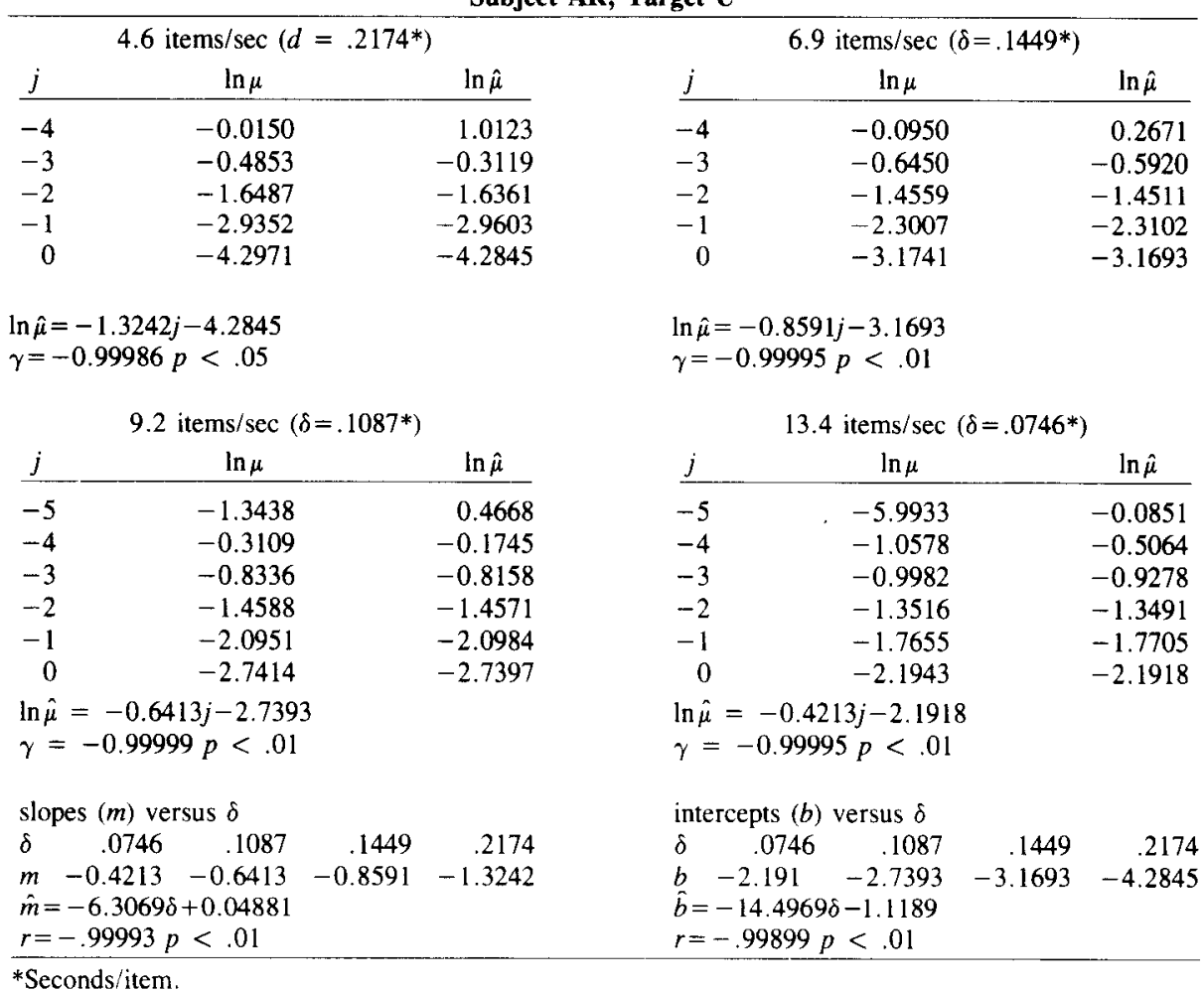

*Seconds/item. 
Table 2

Subject GL, Target C

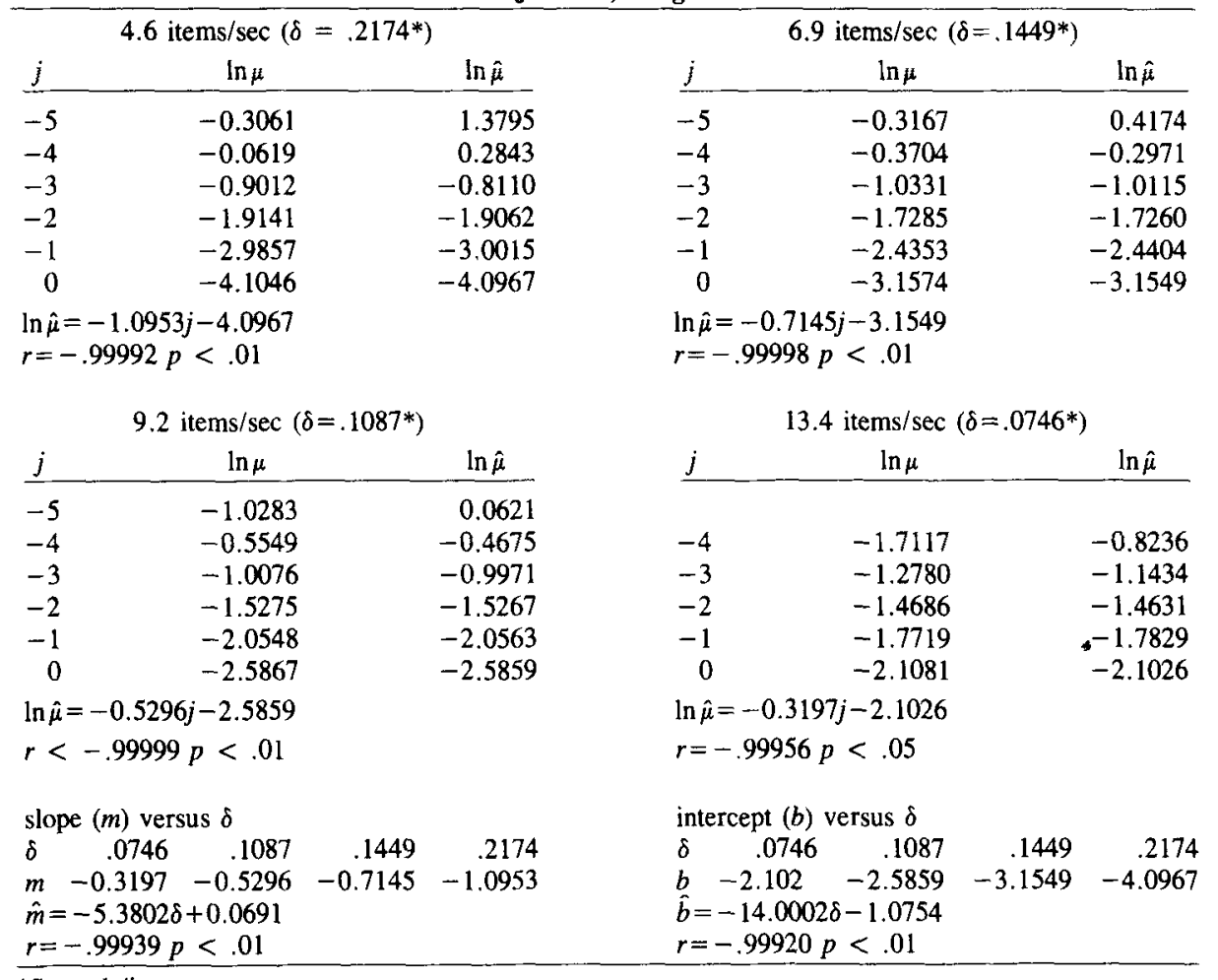

*Seconds/item.

Table 3

Subject AK, Target Square

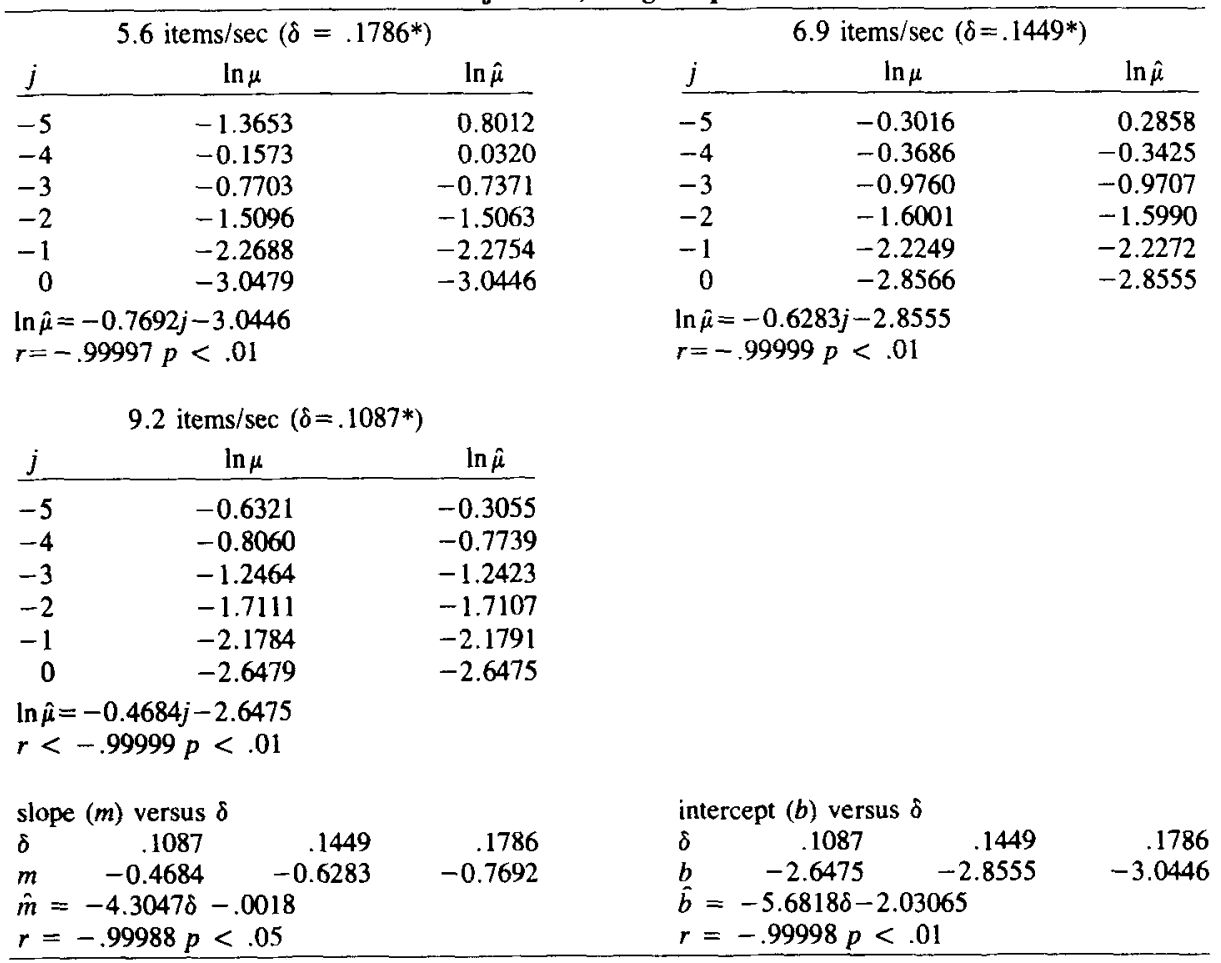

*Seconds/item. 